Geometry $\&$ Topology

Volume 9 (2005) 2261-2302

Published: 6 December 2005

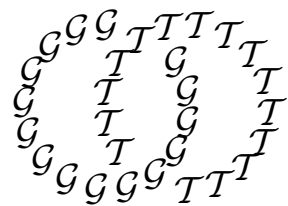

\title{
A splitting formula for the spectral flow of the odd signature operator on 3-manifolds coupled to a path of $S U(2)$ connections
}

\author{
BenJAMin Himpel \\ Mathematisches Institut, Universität Bonn \\ Beringstr. 6, D-53115 Bonn, Germany \\ Email: himpel@math.uni-bonn.de \\ URL: http://www.math.uni-bonn.de/people/himpel/
}

\begin{abstract}
We establish a splitting formula for the spectral flow of the odd signature operator on a closed 3 -manifold $M$ coupled to a path of $S U(2)$ connections, provided $M=S \cup X$, where $S$ is the solid torus. It describes the spectral flow on $M$ in terms of the spectral flow on $S$, the spectral flow on $X$ (with certain Atiyah-Patodi-Singer boundary conditions), and two correction terms which depend only on the endpoints.

Our result improves on other splitting theorems by removing assumptions on the non-resonance level of the odd signature operator or the dimension of the kernel of the tangential operator, and allows progress towards a conjecture by Lisa Jeffrey in her work on Witten's 3-manifold invariants in the context of the asymptotic expansion conjecture [17].
\end{abstract}

\section{AMS Classification numbers Primary: 57M27}

Secondary: 57R57, 53D12, 58J30

Keywords: Spectral flow, odd signature operator, gauge theory, Chern-Simons theory, Atiyah-Patodi-Singer boundary conditions, Maslov index

Proposed: Ronald Stern

Seconded: Peter Teichner, Ronald Fintushel
Received: 4 December 2004

Accepted: 1 November 2005 


\section{Introduction}

This article analyzes the spectral flow of the odd signature operator coupled to a path of $S U(2)$ connections on a 3 manifold $M$ given a decomposition $M=S \cup X$ with $S$ a solid torus.

The question is motivated by Edward Witten's description of certain 3-manifold invariants 26] and Lisa Jeffrey's work [17 on the asymptotic expansion conjecture [25, Conjectures 7.6 and 7.7], particularly [17, Conjecture 5.8] in the case of torus bundles over $S^{1}$.

This work is preceded by Paul Kirk and Erik Klassen [20] where they treat the problem of computing the spectral flow between irreducible flat connections under some restrictions. In this paper these restrictions are removed.

In particular Kirk and Klassen analyzed the spectral flow on a torus bundle over the circle by way of the splitting theorem in [8] applied to a decomposition of the manifold into a solid torus and its complement. They showed that the spectral flow between irreducible, flat connections is $0 \bmod 4$ [20. Theorem 7.5], provided that the kernel of the tangential operator for the odd signature operator on the splitting torus along the path of connections has constant dimension.

Kirk and Klassen proposed [20, Appendix] that it might be possible to always to find a solid torus so that the kernel of the tangential operator has constant dimension. It is shown in Proposition 6.2.1 that this is not always the case. Thus we are forced to deal with the case that the dimension changes.

There have been a lot of general splitting formulas [8, 6, 11, 23, 24]. Unfortunately, for all practical purposes one has to make a couple of technical assumptions in order to apply any of these splitting formulas, namely

(1) that the non-resonance level (see 24]) of the operator in question is zero, and

(2) that the kernel of the tangential operator has constant dimension.

The purpose of this article is to establish a splitting formula in the case where one side is a solid torus without making the above assumptions. We will also show how to apply this tool to explicitly to compute spectral flow.

The main results of this article are the following.

- Section 3 introduces a continuous family of Atiyah-Patodi-Singer boundary conditions $\mathcal{P}^{ \pm}(L)$ for manifolds with torus boundary (Definition 3.2.4 Theorem 3.2.5). 
- In Section 4 we consider a decomposition of a 3 manifold $M=X \cup S$ with $S$ the solid torus and express the spectral flow of the twisted odd signature operator between flat $S U(2)$ connections as the sum of the spectral flow on $S$, the spectral flow on $X$ (with boundary conditions $\mathcal{P}^{ \pm}(L)$ ), and two Maslov triple indices (Theorem 4.3.1).

- Section 5 contains an explicit computation of the spectral flow of the twisted odd signature operator between flat connections on the solid torus with boundary conditions $\mathcal{P}^{ \pm}(L)$ (Theorem 5.3.3).

- In Section [6] we use Theorem 4.3.1 to compute the spectral flow of the twisted odd signature operator between irreducible, flat connections on torus bundles over $S^{1}$ (Theorem 6.3.3). In particular we eliminate the technical assumption used in 20] on the dimension of the kernel of the twisted de Rham operator.

Complete calculations and proofs can be found in the author's thesis [15].

Acknowledgments The results of this article were obtained during the author's Ph.D. studies at Indiana University, Bloomington. The author is grateful for the guidance and support of his advisor and friend Paul Kirk. The comments of the referee were much appreciated. The author thanks the MaxPlanck-Institut in Bonn for their hospitality and financial support while this paper was written.

\section{Preliminaries}

Familiarity with $3,20,23,24$ is useful. However, we introduce all the necessary facts for the convenience of the reader.

\section{$2.1 \quad$ Setup}

For the rest of the article let us assume the following.

(1) The orientation of the torus $T=S^{1} \times S^{1}=\left\{\left(e^{i m}, e^{i l}\right) \mid m, l \in[0,2 \pi)\right\}$ is determined by $d m \wedge d l \in \Omega^{2}(T)$. T is given the product metric with the standard metric on $S^{1}$. The fundamental group $\pi_{1} T$ is the free abelian group generated by $\mu=\left\{\left(e^{i m}, 1\right)\right\}$ and $\lambda=\left\{\left(1, e^{i l}\right)\right\}$. 


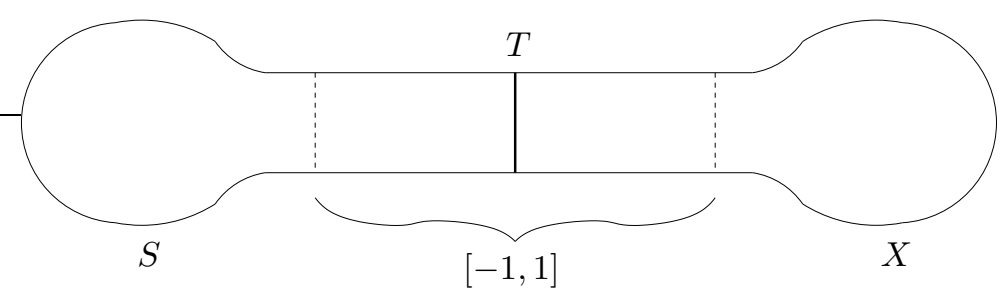

Figure 1: The collar around $T$

(2) The solid torus $S=D^{2} \times S^{1}=\left\{\left(n e^{i m}, e^{i l}\right) \mid n \in[0,1], m, l \in[0,2 \pi)\right\}$ is oriented so that $d n \wedge d m \wedge d l \in \Omega^{3}(S)$ is a positive multiple of the volume form when $n>0$ and we have $\partial S=T$ as oriented manifolds (outward normal first convention). Consider a metric on $D^{2}$ such that a collar of $S^{1}=\partial D^{2}$ may be isometrically identified with $[-1,0] \times S^{1} . S$ is equipped with the product metric of our metrics on $D^{2}$ and $S^{1}$. Then there is a collar $N(T)$ of $\partial S$ which is isometric to $[-1,0] \times T$. This is consistent with our metric on $T$. The fundamental group $\pi_{1} S$ is infinite cyclic generated by $\lambda$, and $\mu$ is trivial in $\pi_{1} S$.

(3) The 3 -manifold $X$ has boundary $T$ and is oriented so that $\partial X=-T$ as oriented manifolds. Consider a metric on $X$ such that a collar of $\partial X$ is isometric to $[0,1] \times T$.

(4) Consider the 3-manifold $M=S \cup_{T} X$ with the orientation and metric induced by the orientation and metric on $S$ and $X$. See Figure 1 . In Section 6, $M$ is a torus bundle over $S^{1}$.

(5) Let $P$ be a trivialized principal bundle with structure group $S U(2)$ over $M$ and consider its restriction to $T, S$ and $X$.

\subsection{Spectral flow}

Let $D_{t}, t \in[0,1]$, be a 1 -parameter family of (possibly unbounded) self-adjoint operators with compact resolvent, continuous in the graph metric. Then, given $\varepsilon>0$ smaller than the modulus of the largest negative eigenvalue of $D_{0}$ and $D_{1}$, the spectral flow $\mathrm{SF}\left(D_{t}\right) \in \mathbf{Z}$ is roughly defined to be the algebraic intersection number in $[0,1] \times \mathbf{R}$ of the track of the spectrum

$$
\left\{(t, \lambda) \mid t \in[0,1], \lambda \in \operatorname{Spec}\left(D_{t}\right)\right\}
$$

and the line segment from $(0,-\varepsilon)$ to $(1,-\varepsilon)$. The orientations are chosen so that if $D_{t}$ has spectrum $\{n+t \mid n \in Z\}$ then $\operatorname{SF}\left(D_{t}\right)=1$. For a detailed discussion on spectral flow with rigorous definitions and proofs see 4 . 
Given a continuous family $D_{t}$ of self-adjoint elliptic operators on a closed manifold $M$, one can use standard theorems (for example in [18]) to conclude that the spectrum is discrete for each $t$ and varies continuously in $t$.

For a compact manifold $Y$ with boundary $\Sigma$ one needs to choose boundary conditions. In [4] the authors treat this in more generality, but for the results in the present article it is sufficient to confine ourselves to Atiyah-PatodiSinger boundary conditions (see Section 2.5 for a definition) on a manifold with a collar. It is shown in 21, that by choosing a continuous family of AtiyahPatodi-Singer boundary conditions the spectrum of $D_{t}$ varies continuously in $t$ (see [21, Theorem 4.1 and Section 5]).

Observe, that there are other conventions for spectral flow (see for example [17. and [20]). However, they differ only by $\operatorname{dim}\left(\operatorname{Ker}\left(D_{0}\right)\right)$ or $\operatorname{dim}\left(\operatorname{Ker}\left(D_{1}\right)\right)$.

\subsection{Connections and representations}

There are several equivalent definitions for connections, but in the case of principal $S U(2)$ bundles over 3 -manifolds we have a particularly nice description for connections.

We will identify the Lie group $S U(2)$ with the unit quaternions

$$
\{v \in \mathbf{R} \oplus \mathbf{R} i \oplus \mathbf{R} j \oplus \mathbf{R} k|| v \mid=1\} .
$$

The group multiplication will be the usual multiplication in the quaternions. Its Lie algebra $s u(2)$ can be identified with the vector space $\mathbf{R} i \oplus \mathbf{R} j \oplus \mathbf{R} k=\mathbf{R} i \oplus \mathbf{C} j$ of imaginary quaternions. The adjoint action ad of $S U(2)$ on $s u(2)$ corresponds to the conjugation of imaginary quaternions by unit quaternions.

Consider the principal $S U(2)$ bundle $P$ over $M$. The chosen trivialization of $P$ induces an identification

- of the group of gauge transformations with $\mathcal{G}_{M}:=C^{\infty}(M, S U(2))$, and

- of the affine space of connections with Lie-algebra-valued 1-forms $\mathcal{A}_{M}:=$ $\Omega^{1}(M) \otimes s u(2)$ on $M$, where the product (or trivial) connection corresponds to $0 \in \Omega^{1}(M) \otimes s u(2)$.

Then the action of a gauge transformation $g \in C^{\infty}(M, S U(2))$ on an $S U(2)$ connection $A \in \Omega^{1}(M) \otimes s u(2)$ on $M$ is given by $g \cdot A=g A g^{-1}+g d g^{-1}$.

The choice of trivialization $P=M \times S U(2)$ induces a trivialization of the adjoint bundle $P \times{ }_{\text {ad }} s u(2)$ associated to $P$. Thus we can associate to a connection 
$A$ on $P$ a covariant derivative on $\operatorname{ad} P=M \times s u(2)$ by defining

$$
\begin{aligned}
d_{A}: \Omega^{0}(M) \otimes s u(2) & \rightarrow \Omega^{1}(M) \otimes s u(2) \\
a & \mapsto d a+[A, a],
\end{aligned}
$$

where $[\cdot, \cdot]$ means taking the Lie bracket on the coefficients and wedging the form part. We extend the covariant derivative to $\Omega^{*}(M) \otimes s u(2)$ by the Leibniz rule.

The curvature $F_{A}$ of a connection $A \in \Omega^{1}(M) \otimes s u(2)$ is the 2 -form in $\Omega^{2}(M) \otimes$ $s u(2)$ defined by $\left[F_{A}, \varphi\right]:=d_{A}^{2} \varphi$, where $\varphi \in \Omega^{0}(M) \otimes s u(2)$. We have $F_{A}=$ $d A+A \wedge A$. We call $A$ flat, if $F_{A}=0$. Let $\mathcal{F}_{M} \subset \mathcal{A}_{M}$ be the set of all flat connections on $M$. If $A$ is flat, then the holonomy hol $(A): \pi_{1} M \rightarrow S U(2)$ is a $S U(2)$ representation of the fundamental group $\pi_{1} M$ of $M$.

Let $\chi_{M}$ be the set of conjugacy classes of $S U(2)$ representations of the fundamental group $\pi_{1}(M)$. Then the holonomy map identifies $\mathcal{F}_{M} / \mathcal{G}_{M}$ with $\chi_{M}$ 13. Proposition 2.2.3].

\subsection{The odd signature operator and the de Rham operator}

We introduce two first order differential operators on $M$ and $T$. These depend on the orientation and the Riemannian metric on $M$ and $T$, which we fixed in Section 2.1] We set $\Omega^{i}(M ; s u(2)):=\Omega^{i}(M) \otimes s u(2), \Omega^{0+1}(M ; s u(2)):=$ $\Omega^{0}(M ; s u(2)) \oplus \Omega^{1}(M ; s u(2))$.

The $L^{2}$ inner product on $\Omega^{0+1}(M ; s u(2))$ and $\Omega^{0+1+2}(T ; s u(2))$ is given in terms of the Hodge $*$ operator by

$$
\langle\alpha, \beta\rangle_{L^{2}(M)}=-\int_{M} \operatorname{tr}(\alpha \wedge * \beta) \quad \text { and } \quad\langle\alpha, \beta\rangle_{L^{2}(T)}=-\int_{T} \operatorname{tr}(\alpha \wedge * \beta) .
$$

For an $S U(2)$ connection $A \in \Omega^{1}(M ; s u(2))$ the odd signature operator twisted by $A$ is defined to be

$$
\begin{aligned}
D_{A}: \Omega^{0+1}(M ; s u(2)) & \rightarrow \Omega^{0+1}(M ; s u(2)) \\
(\alpha, \beta) & \mapsto\left(d_{A}^{*} \beta, * d_{A} \beta+d_{A} \alpha\right) .
\end{aligned}
$$

For an $S U(2)$ connection $a \in \Omega^{1}(T ; s u(2))$ de Rham operator twisted by $a$ is defined to be

$$
\begin{aligned}
S_{a}: \Omega^{0+1+2}(T ; s u(2)) & \rightarrow \Omega^{0+1+2}(T ; s u(2)) \\
(\alpha, \beta, \gamma) & \mapsto\left(* d_{a} \beta,-* d_{a} \alpha-d_{a} * \gamma, d_{a} * \beta\right) .
\end{aligned}
$$


In fact, $S_{a}$ is the tangential operator of $D_{A}$ (see [3, Lemma 2.4]). $D_{A}$ and $S_{a}$ are first-order self-adjoint elliptic differential operator.

The following important and well-known fact is an application of the AtiyahPatodi-Singer index theorem (compare with [22, Theorem 7.1]):

Proposition 2.4.1 Suppose $M$ is a closed 3-manifold and $g: M \rightarrow S U(2)$ is a gauge transformation. If $A_{t}$ is any path of $S U(2)$ connections on $M$ from $A_{0}$ to $A_{1}=g \cdot A_{0}$, then

$$
\mathrm{SF}\left(D_{A_{t}}\right)=8 \operatorname{deg}(g)
$$

\subsection{Eigenspaces of the de Rham operator}

Let $a \in \mathcal{A}_{T}$ and $\nu \geq 0$. Denote by $E_{a, \nu}$ the $\nu$-eigenspace of $S_{a}$ and let

$$
\begin{aligned}
& P_{a, \nu}^{+}:=\operatorname{span}_{L^{2}}\left\{\psi \mid S_{a} \psi=\mu \psi, \mu>\nu\right\}=\bar{\bigoplus}_{\mu>\nu} E_{a, \mu}{ }^{2}, \\
& P_{a, \nu}^{-}:=\operatorname{span}_{L^{2}}\left\{\psi \mid S_{a} \psi=\mu \psi, \mu<-\nu\right\}=\bar{\bigoplus}_{\mu<-\nu} E_{a, \mu} L^{2} \\
& E_{a, \nu}^{+}:=\bigoplus_{0<\mu \leq \nu} E_{a, \mu} \text { and } \\
& E_{a, \nu}^{-}:=\bigoplus_{-\nu \leq \mu<0} E_{a, \mu} .
\end{aligned}
$$

Abbreviate $P_{a}^{ \pm}:=P_{a, 0}^{ \pm}$. By the spectral theorem for self-adjoint elliptic operators we have

$$
L^{2}\left(\Omega^{0+1+2}(T, s u(2))\right)=P_{a}^{+} \oplus \operatorname{Ker} S_{a} \oplus P_{a}^{-} .
$$

We define an almost complex structure on $L^{2}\left(\Omega^{0+1+2}(T ; s u(2))\right)$ by

$$
J(\alpha, \beta, \gamma):=(-* \gamma, * \beta, * \alpha) .
$$

One can see that $J^{2}=-\mathrm{Id}$ and that $J$ is an isometry of $L^{2}\left(\Omega^{0+1+2}(T ; s u(2))\right)$. The induced symplectic structure $\omega(x, y):=\langle x, J y\rangle$ is compatible with $J$, which makes $L^{2}\left(\Omega^{0+1+2}(T ; s u(2))\right)$ into a symplectic space with compatible almost complex structure. A subspace $\Lambda$ of $L^{2}\left(\Omega^{0+1+2}(T ; s u(2))\right)$ is Lagrangian if $J \Lambda=\Lambda^{\perp}$.

$\operatorname{Ker} S_{a}$ is a finite dimensional symplectic subspace of $L^{2}\left(\Omega^{0+1+2}(T ; s u(2))\right)$. Furthermore, if $L$ is a Lagrangian subspace of $\operatorname{Ker} S_{a}$, then $L \oplus P_{a}^{ \pm}$is Lagrangian in $L^{2}\left(\Omega^{0+1+2}(T ; s u(2))\right)$. 
A Lagrangian subspace $\mathcal{P} \subset L^{2}\left(\Omega^{0+1+2}(\Sigma ; s u(2))\right)$ is called an Atiyah-PatodiSinger (APS) boundary condition, if $\mathcal{P}$ contains all eigenvectors of the tangential operator $S_{a}$ with sufficiently large eigenvalues.

Let $\Delta_{a}:=d_{a} d_{a}^{*}+d_{a}^{*} d_{a}$ be the Laplacian on $\Omega^{0+1+2}(T ; s u(2))$ twisted by $a \in \mathcal{A}_{T}$. Denote the harmonic 0,1 and 2 -forms of $\Delta$ and $\Delta_{a}$ by $\mathcal{H}^{0+1+2}(T ; s u(2)):=$ $\operatorname{Ker} \Delta$ and $\mathcal{H}_{a}^{0+1+2}(T ; s u(2)):=\operatorname{Ker} \Delta_{a}$ respectively.

If $a$ is flat, we have $d_{a}^{2}=0$ and consequently $\Delta_{a}=S_{a}^{2}$. Thus a $\lambda$-eigenvector $\varphi$ of $S_{a}$ is a $\lambda^{2}$-eigenvector of $\Delta_{a}$. Furthermore, a direct computation shows the following.

Lemma 2.5.1 If $a$ is flat and $\varphi$ is a $\lambda^{2}$-eigenvector $(\lambda>0)$ for $\Delta_{a}$, then $\varphi \pm \frac{1}{\lambda} S_{a} \varphi$ is a $\pm \lambda$-eigenvector for $S_{a}$. Furthermore $\operatorname{Ker} S_{a}=\mathcal{H}_{a}^{0+1+2}(T ; s u(2))$.

\subsection{Cauchy data spaces and adiabatic limits}

Cauchy data spaces play an important role in this work because of their relation to spectral flow. Liviu Nicolaescu analyzed this relationship in 24. His results have been extended by [1] and [23]. The facts in this section make up the main tools for this work.

We will state all results in this section in terms of the odd signature operator on $M$, but they apply to other self-adjoint Dirac type operators as well.

We introduce the notation

$$
\begin{array}{ccl}
S^{R}=S \cup([0, R] \times T) & \text { and } & S^{\infty}=S \cup([0, \infty) \times T), \\
X^{R}=X \cup([-R, 0] \times T) & \text { and } & X^{\infty}=X \cup((-\infty, 0] \times T)
\end{array}
$$

for $R \geq 0$.

Let $A$ be a connection on $X$, which is in cylindrical form in a collar of $T$, that is $A=i_{u}^{*} a$, where $i_{u}: T \hookrightarrow[-1,1] \times T$ is the inclusion at $u \in[-1,1]$ and $a \in \mathcal{A}_{T}$.

We write the restriction of $\Omega^{0+1}([-1,1] \times T ; s u(2))$ to $T$ as

$$
\begin{aligned}
r: \Omega^{0+1}([-1,1] \times T ; s u(2)) & \rightarrow \Omega^{0+1+2}(T ; s u(2)) \\
(\sigma, \tau) & \left.\mapsto\left(i_{0}^{*}(\sigma), i_{0}^{*}(\tau), * i_{0}^{*}(\tau\lrcorner \frac{\partial}{\partial u}\right)\right),
\end{aligned}
$$

where $\tau\lrcorner \frac{\partial}{\partial u}$ denotes contraction of $\tau$ with $\frac{\partial}{\partial u}$, and $*$ is the Hodge star on differential forms on the 2 -manifold $T$. This also gives us a restriction map of 
$\Omega^{0+1}(S ; s u(2))$ and $\Omega^{0+1}(X ; s u(2))$ to $\Omega^{0+1+2}(T ; s u(2))$. If we write $\tau=\beta+$ $\omega d u$, where $u$ is the coordinate in $[0,1]$ and $\beta$ does not have a $d u$ component, then a more intuitive way to write the restriction map is $r(\sigma, \beta+\omega d u)=$ $\left(\left.\sigma\right|_{T},\left.\beta\right|_{T}, *\left(\left.\omega\right|_{T}\right)\right)$.

The Cauchy data space of $D_{A}$ is

$$
\Lambda_{X, A}:=\Lambda_{X}\left(D_{A}\right):={\overline{r\left(\operatorname{Ker} D_{A}\right)}}^{L^{2}},
$$

and the scattering Lagrangian or the limiting values of extended $L^{2}$ solutions is

$$
\mathscr{L}_{X, A}:=\operatorname{proj}_{\operatorname{Ker} S_{a}}\left(\Lambda_{X, A} \cap\left(P_{a}^{-} \cup \operatorname{Ker} S_{a}\right)\right) .
$$

See 2] for more information on Cauchy data spaces, particularly 2], Definition 2.22]. Note that we can extend $D_{A}$ to $X^{R}$ and that $\Lambda_{X, A}^{R}:=\Lambda_{X}\left(D_{A}\right)$ is a continuous family of Lagrangian subspaces by [11, Lemma 3.2]. Denote $\Lambda_{X, A}^{\infty}:=$ $\lim _{R \rightarrow \infty} \Lambda_{X, A}^{R}$.

For $a$ flat the kernel of $S_{a}$ is isomorphic to the cohomology $H^{*}\left(\partial X ; s u(2)_{\operatorname{hol}(a)}\right)$ with values in $s u(2)$ twisted by $\operatorname{hol}(a)$ via the Hodge and de Rham theorems. See for example [12, Chapter 5] for a definition of the cohomology of $X$ twisted by a representation $\rho: \pi(X) \rightarrow \operatorname{Aut}(V)$.

The following gives a way to compute the scattering Lagrangian at a flat connection.

Proposition 2.6.1 (Corollary 8.4, 23]) If $A$ is flat on $X$, then $\mathscr{L}_{X, A}$ is isomorphic to $\operatorname{Im}\left(H^{*}\left(X ; s u(2)_{\operatorname{hol}(A)}\right) \rightarrow H^{*}\left(\partial X ; s u(2)_{\operatorname{hol}(A)}\right)\right)$ via the Hodge and de Rham theorems.

Cauchy data spaces are complicated and we wish to relate them to simpler Lagrangians. The situation is particularly favorable when $D_{A}$ has non-resonance level 0. Nicolaescu [24] defines $D_{A}$ to have non-resonance level $\nu \geq 0$, if $P_{a, \nu}^{-} \cap \Lambda_{X, A}=0$.

Nicolaescu's adiabatic limit theorem describes the limit of the Cauchy data spaces of $D_{A}$ under stretching the collar.

Theorem 2.6.2 (Corollary 4.11, 24]) If $D_{A}$ has non-resonance level 0, then

$$
\Lambda_{X, A}^{\infty}=P_{a}^{+} \oplus \mathscr{L}_{X, A}
$$

We will need some facts about the 0 non-resonance level situation. In analogy to [3, Proposition 2.10] we have the following from [1, Proposition 4.9]. 
Proposition 2.6.3 Let $A$ be flat.

(1) We have

$$
\Lambda_{X, A} \cap P_{a}^{-} \cong \operatorname{Im}\left(H^{1}\left(X, T ; s u(2)_{\operatorname{hol}(A)}\right) \rightarrow H^{1}\left(X ; s u(2)_{\operatorname{hol}(A)}\right)\right) .
$$

Thus 0 non-resonance level is equivalent to

$$
\operatorname{Im}\left(H^{1}\left(X, T ; s u(2)_{\operatorname{hol}(A)}\right) \rightarrow H^{1}\left(X ; s u(2)_{\operatorname{hol}(A)}\right)\right)=0 .
$$

(2) Assuming 0 non-resonance level, we get the isomorphism

$$
\Lambda_{X, A} \cap\left(P_{a}^{-} \oplus Q\right) \cong \mathscr{L}_{X} \cap Q .
$$

Kirk and Lesch 23] give a detailed decomposition of $L^{2}\left(\Omega^{0+1+2}(T ; s u(2))\right)$ in the spirit of the Hodge decomposition as an orthogonal sum of symplectic spaces

$$
\left(P_{a, \nu}^{-} \oplus\left(P_{a, \nu}^{+}\right)\right) \oplus\left(d_{a}\left(E_{a, \nu}^{+}\right) \oplus d_{a}^{*}\left(E_{a, \nu}^{-}\right)\right) \oplus\left(d_{a}^{*}\left(E_{a, \nu}^{+}\right) \oplus d_{a}\left(E_{a, \nu}^{-}\right)\right) \oplus \operatorname{Ker} S_{a} \text {. (2.6.1) }
$$

When $A$ is flat, we do not only get an explicit description of the scattering Lagrangian (Proposition 2.6.1), but the adiabatic limit has a nice description when the the non-resonance level is not 0 .

Theorem 2.6.4 (Theorem 8.5, 23]) If $A$ is flat, then there exists a subspace

$$
W_{a} \subset d_{a}\left(E_{a, \nu}^{+}\right) \subset P_{a, 0}^{-}
$$

isomorphic to

$$
\operatorname{Im}\left(H^{0+1}\left(X, T ; s u(2)_{\operatorname{hol}(A)}\right) \rightarrow H^{0+1}\left(X ; s u(2)_{\operatorname{hol}(A)}\right)\right)
$$

so that if $W_{a}^{\perp}$ denotes the orthogonal complement of $W_{a}$ in $d_{a}\left(E_{a, \nu}^{+}\right)$, then with respect to the decomposition (2.6.1) into symplectic subspaces, the adiabatic limit of the Cauchy data spaces decomposes as a direct sum of Lagrangian subspaces:

$$
\Lambda_{X, A}^{\infty}=P_{a, \nu}^{+} \oplus\left(W_{a} \oplus J\left(W_{a}^{\perp}\right)\right) \oplus d_{a}\left(E_{a, \nu}^{-}\right) \oplus \mathscr{L}_{A}
$$

where $\mathscr{L}_{A} \subset \operatorname{Ker} S_{a} \cong H^{*}\left(T ; s u(2)_{\operatorname{hol}(A)}\right)$ denotes the scattering Lagrangian on $X$.

In due course, we will need to have control over the intersection of the Cauchy data spaces with other Lagrangian subspaces when we stretch the collar. Thus the following results are important.

Proposition 2.6.5 (Lemma 8.10, 23]) Let $A$ be flat, and consider a Lagrangian subspace $V \subset \operatorname{Ker} S_{a}$.

(1) The dimension of $\Lambda_{S, A}^{R} \cap \Lambda_{X, A}^{R}$ is independent of $R \in[0, \infty]$.

(2) The dimension of $\Lambda_{S, A}^{R} \cap\left(P_{a}^{+} \oplus V\right)$ is independent of $R \in[0, \infty]$.

(3) The dimension of $\left(P_{a}^{-} \oplus V\right) \cap \Lambda_{X, A}^{R}$ is independent of $R \in[0, \infty]$. 


\subsection{Maslov index}

Let $H$ be a symplectic Hilbert space with compatible almost complex structure $J$. A pair of Lagrangians $(L, M)$ in $H$ is called Fredholm if $L+M$ is closed and both $\operatorname{dim}(L \cap M)$ and $\operatorname{codim}(L+M)$ are finite. Consider a continuous path $\left(L_{t}, M_{t}\right)$ of Fredholm pairs of Lagrangians in $H$. Continuity is measured in the gap topology. If $L_{t}$ and $M_{t}$ are transverse at the end points, that is, intersect trivially, then the Maslov index $\operatorname{Mas}\left(L_{t}, M_{t}\right)$ is roughly defined to be a count of how many times $L_{t}$ and $M_{t}$ intersect with sign and multiplicity, that is, counting the dimension of the intersection. For a careful definition see 7, 24, 9]. If the intersection of $L_{t}$ and $M_{t}$ is nontrivial at the endpoints, we will choose a convention compatible with our spectral flow convention (in view of Theorem 2.7.1): Given a continuous 1-parameter family of Fredholm pairs of Lagrangians $\left(L_{t}, M_{t}\right), t \in[0,1]$, choose $\varepsilon>0$ small enough so that

(1) $e^{s J} L_{i}$ is transverse to $M_{i}$ for $i=0,1$ and $0<s \leq \varepsilon$, and

(2) $\left(e^{s J} L_{t}, M_{t}\right)$ is a Fredholm pair for all $t \in[0,1]$ and all $0 \leq s \leq \varepsilon$.

Then the Maslov index of the pair $\left(L_{t}, M_{t}\right)$ is the Maslov index of $\left(e^{\varepsilon L} L_{t}, M_{t}\right)$.

A splitting theorem for spectral flow by Nicolaescu 24] in terms of a Maslov index has been extended in [10] to the situation when the Dirac operators are not invertible at the endpoints. Also see [23, Theorem 7.6] for a proof of the same result. The precise statement in the context of the odd signature operator and $S U(2)$ connections on $M=S \cup_{T} X$ is the following.

Theorem 2.7.1 (Theorem 4.3, 10]) Suppose $A_{t}$ is a continuous path of $S U(2)$ connections on $M$ in cylindrical form in a collar of $T$. Then the path $\left.\left(\Lambda_{S}\left(D_{A_{t}}\right)\right), \Lambda_{X}\left(D_{A_{t}}\right)\right)$ consists of Fredholm pairs of Lagrangians and

$$
\operatorname{SF}\left(D_{A_{t}}\right)=\operatorname{Mas}\left(\Lambda_{S}\left(D_{A_{t}}\right), \Lambda_{X}\left(D_{A_{t}}\right)\right) .
$$

We also have a relative version of this theorem (see 9] and [24]) which relates spectral flow on a manifold with boundary with APS boundary conditions to some Maslov index. It is implied by the results in [10].

Theorem 2.7.2 If $A_{t}$ is a path of connections on $X$ in cylindrical form near $T$ and $\mathcal{P}_{t}$ is a continuous family of self-adjoint APS boundary conditions, then the spectral flow $\operatorname{SF}\left(D_{A_{t}} \mid X ; \mathcal{P}_{t}\right)$ is well defined and $\operatorname{SF}\left(D_{A_{t}} \mid X ; \mathcal{P}_{t}\right)=$ $\operatorname{Mas}\left(\Lambda_{X}\left(D_{A_{t}}\right), \mathcal{P}_{t}\right)$. 
We will also use a Maslov triple index as defined in [23, which is up to normalization the same as Bunke's Maslov triple index in [6] and different from the Maslov triple index $\tau_{H}$ usually considered in the literature (see [7]). In our notation, we define it for triples $\left(L_{1}, L_{2}, L_{3}\right)$ of Lagrangian subspaces of a symplectic Hilbert space with almost complex structure $J$, such that $\left(J L_{i}, L_{j}\right)$ is a Fredholm pair for all $i, j=1,2,3$. We set $\tau_{\mu}(L, L, L):=0$ for some Lagrangian subspace $L$ and use [23, Formula (6.21)] to define $\tau_{\mu}$ for other triples: If $L_{1, t}, L_{2, t}$ and $L_{3, t}, t \in[0,1]$ are paths of Lagrangian subspaces, such that $\left(J L_{i, t}, L_{j, t}\right)$ is a Fredholm pair for all $i, j=1,2,3, t \in[0,1]$, then the (twisted) Maslov triple index $\tau_{\mu}$ is determined by requiring that

$$
\begin{aligned}
& \tau_{\mu}\left(L_{1,1}, L_{2,1}, L_{3,1}\right)-\tau_{\mu}\left(L_{1,0}, L_{2,0}, L_{3,0}\right) \\
& \quad=\operatorname{Mas}\left(J L_{1}, L_{2}\right)+\operatorname{Mas}\left(J L_{2}, L_{3}\right)-\operatorname{Mas}\left(J L_{1}, L_{3}\right) .
\end{aligned}
$$

The indices $\tau_{\mu}$ and $\tau_{H}$ share some properties. For example $\tau_{\mu}$ is additive under direct sums (symplectic additivity). Furthermore the following properties are an elementary consequence of the above characterization, and can also be found in 23, Proposition 6.11].

Lemma 2.7.3 Let $L_{i}, i=1, \ldots, 4$ be pairwise Fredholm Lagrangians in a Hilbertspace $H$. Then

- $\tau_{\mu}\left(L_{1}, L_{1}, L_{2}\right)=\tau_{\mu}\left(L_{1}, L_{2}, L_{2}\right)=0$, and

- $\tau_{\mu}\left(L_{1}, L_{2}, L_{1}\right)=\operatorname{dim}\left(J L_{1} \cap L_{2}\right)$.

- $\tau_{\mu}\left(L_{1}, L_{2}, L_{3}\right)=\operatorname{dim}\left(J L_{2} \cap L_{3}\right)-\tau_{\mu}\left(L_{1}, L_{3}, L_{2}\right)$.

\section{A family of Atiyah-Patodi-Singer boundary con- ditions}

This section introduces a specific family of Atiyah-Patodi-Singer boundary conditions for the odd signature operator on a manifold with torus boundary.

The results will be used for the analysis of the spectral flow for the splitting $M=X \cup_{T} S$ in Section [4 where $S$ is the solid torus. In Section, 5] we will explicitly compute spectral flow on $S$ with the boundary conditions developed in this section. 


\subsection{A family of flat connections on the torus}

Let $\chi_{T}=\operatorname{Hom}\left(\pi_{1} T, S U(2)\right) /$ conj be the set of conjugacy classes of $S U(2)$ representations of the fundamental group $\pi_{1} T$ of $T$.

The holonomy map gives a homeomorphism from the gauge equivalence classes of the flat $S U(2)$ connections $\mathcal{F}_{T} / \mathcal{G}_{T}$ on $T$ to $\chi_{T}$ [13, Proposition 2.2.3]. If $A=$ $-i \alpha d m-i \beta d l$ with $(\alpha, \beta) \in \mathbf{R}^{2}$, then $\operatorname{hol}(A)=\rho_{(\alpha, \beta)}$ is given in quaternionic notation by

$$
\begin{aligned}
\rho_{(\alpha, \beta)}: \pi_{1}(T) & \rightarrow S U(2) \\
\mu & \mapsto e^{2 \pi i \alpha} \\
\lambda & \mapsto e^{2 \pi i \beta} .
\end{aligned}
$$

Notice, that $\mathbf{R}^{2} \rightarrow \chi_{T},(\alpha, \beta) \rightarrow\left[\rho_{(\alpha, \beta)}\right]$ is a branched cover of $\chi_{T}$, with branch points the half integer lattice, which map to central representations, and covering transformations $(\alpha, \beta) \rightarrow( \pm \alpha+m, \pm \beta+n),(m, n) \in \mathbf{Z}^{2}$. Each $(\alpha, \beta)$ then also corresponds to an $S U(2)$ connection $-i \alpha d m-i \beta d l$ on $T$. Thus, We have a smooth family $\{-i \alpha d m-i \beta d l\}$ of flat connections with holonomy $\rho_{(\alpha, \beta)}$ as in (3.1.1) parametrized by $\mathbf{R}^{2}$, which projects onto $\mathcal{F}_{T} / \mathcal{G}_{T}=\chi_{T}$.

Definition 3.1.1 Let $a_{\alpha, \beta}:=-i \alpha d m-i \beta d l$. We substitute an index $a_{\alpha, \beta}$ by $(\alpha, \beta)$, for example $\mathcal{H}_{\alpha, \beta}^{0}(T ; s u(2))=\mathcal{H}_{a_{\alpha, \beta}}^{0}(T ; s u(2))$ and $S_{\alpha, \beta}=S_{a_{\alpha, \beta}}$.

The harmonic forms of $\Delta_{\alpha, \beta}=\Delta_{a_{\alpha, \beta}}$ are equal to the kernel of $S_{\alpha, \beta}$ by Lemma 2.5.1. By $e^{i(2 \alpha m+2 \beta l)} \mathbf{C} j$ we denote the $\mathbf{C}$-linear combinations of the function $e^{i(2 \alpha m+2 \beta l)} j$. The following straightforward computation of the harmonic forms on the torus is left to the reader.

\section{Proposition 3.1.2}

$$
\begin{aligned}
& \mathcal{H}_{\alpha, \beta}^{0}(T ; s u(2))= \begin{cases}\mathbf{R} i & \text { for }(\alpha, \beta) \notin\left(\frac{1}{2} \mathbf{Z}\right)^{2}, \\
\mathbf{R} i \oplus e^{i(2 \alpha m+2 \beta l)} \mathbf{C} j & \text { for }(\alpha, \beta) \in\left(\frac{1}{2} \mathbf{Z}\right)^{2}\end{cases} \\
& \mathcal{H}_{\alpha, \beta}^{1}(T ; s u(2))= \begin{cases}\mathbf{R} i d m \oplus \mathbf{R} i d l & \text { for }(\alpha, \beta) \notin\left(\frac{1}{2} \mathbf{Z}\right)^{2}, \\
\left\{\begin{array}{l}
\mathbf{R} i d m \oplus e^{i(2 \alpha m+2 \beta l)} \mathbf{C} j d m \\
\oplus \mathbf{R} i d l \oplus e^{i(2 \alpha m+2 \beta l)} \mathbf{C} j d l
\end{array}\right\} & \text { for }(\alpha, \beta) \in\left(\frac{1}{2} \mathbf{Z}\right)^{2}\end{cases} \\
& \mathcal{H}_{\alpha, \beta}^{2}(T ; s u(2)) \cong \mathcal{H}_{\alpha, \beta}^{0}(T ; s u(2)) \text { via the Hodge star. }
\end{aligned}
$$

Also observe that $S_{\alpha, \beta}$ preserves the $\mathbf{R} i$ and $\mathbf{C} j$-part of $L^{2}\left(\Omega^{0+1+2}(T ; s u(2))\right)$, and we can write

$$
P_{\mathbf{R} i}^{ \pm}=P_{\alpha, \beta}^{ \pm} \cap L^{2}\left(\Omega^{0+1+2}(T ; \mathbf{R} i)\right) \text { and } P_{\alpha, \beta, \mathbf{C} j}^{ \pm}=P_{\alpha, \beta}^{ \pm} \cap L^{2}\left(\Omega^{0+1+2}(T ; \mathbf{C} j)\right) .
$$


as well as

$$
\mathcal{H}^{0+1+2}(T ; \mathbf{R} i)=\mathcal{H}_{\alpha, \beta}^{0+1+2}(T ; \mathbf{R} i)
$$

\subsection{Boundary conditions}

We want a path of self-adjoint operators which is continuous in the graph topology. In order for the odd signature operator $D_{A_{t}}$ on $S$ or $X$ to vary continuously and be (unbounded) self-adjoint, we need a path of boundary conditions which is continuous in the gap topology. See 4 for details.

We might like to pick a continuous family of Lagrangians in $\mathcal{H}_{\alpha, \beta}^{0+1+2}(T ; s u(2))$ and extend it to a family of APS boundary conditions in $L^{2}\left(\Omega^{0+1+2}(T ; s u(2))\right)$ by $P_{\alpha, \beta}^{+}$or $P_{\alpha, \beta}^{-}$. Unfortunately, not only does the dimension of the kernel of the tangential operator jump up at $\left(\frac{1}{2} \mathbf{Z}\right)^{2}$ (see Proposition [3.1.2), but for a smooth path $\varrho_{t}, t \in[-1,1]$, through $\varrho_{0} \in\left(\frac{1}{2} \mathbf{Z}\right)^{2}$ we have

$$
\lim _{t \rightarrow 0^{+}} P_{\varrho t}^{ \pm} \neq \lim _{t \rightarrow 0^{-}} P_{\varrho t}^{ \pm} .
$$

In fact the $\mathcal{H}_{\alpha, \beta}^{0+1+2}(T ; \mathbf{C} j)$ part of the limits turn out to be orthogonal to each other.

Thus we introduce the space $\dot{\mathbf{R}}^{2}$ shown in Figure 2 to parametrize the Lagrangians. It is $\mathbf{R}^{2}$ with open disks of radius $\frac{1}{8}$ removed around all half integer lattice points with the induced topology. Some people would call it the real blow-up of the plane at the half integer lattice points. We will see in Theorem 3.2.2. why this is a good parameter space. The advantage of this space is that we can easily homotop paths of connections together with their boundary conditions, thus getting a homotopy of paths of self-adjoint operators. However, it will be more convenient to parametrize $\dot{\mathbf{R}}^{2}$ by the following space.

Definition 3.2.1 Let $\widetilde{\mathbf{R}}^{2}:=\mathbf{R}^{2} \times S^{1} / \sim$, where $(\alpha, \beta, \theta) \sim(\alpha, \beta, 1)$ if $(\alpha, \beta) \notin$ $\left(\frac{1}{2} \mathbf{Z}\right)^{2}$. We will simply write $(\alpha, \beta, \theta) \in \widetilde{\mathbf{R}}^{2}$. Alternatively it is convenient to think of elements in $\widetilde{\mathbf{R}}^{2}$ as being of the form

$$
\begin{cases}(\alpha, \beta, \theta) & \text { if }(\alpha, \beta) \in\left(\frac{1}{2} \mathbf{Z}\right)^{2} \text { and } \theta \in S^{1}, \\ (\alpha, \beta) & \text { if }(\alpha, \beta) \notin\left(\frac{1}{2} \mathbf{Z}\right)^{2} .\end{cases}
$$

Denote by $\pi: \widetilde{\mathbf{R}}^{2} \rightarrow \mathbf{R}^{2}$ the projection $(\alpha, \beta, \theta) \rightarrow(\alpha, \beta)$. Let us define a bijection $h$ between $\widetilde{\mathbf{R}}^{2}$ and $\dot{\mathbf{R}}^{2}$.

We will describe what this bijection looks like around the origin. At all the other half integer lattice points we get a similar bijection via translation. Away 


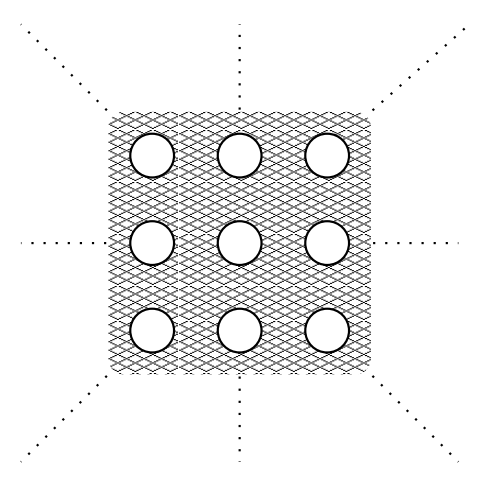

Figure $2: \dot{\mathbf{R}}^{2} \approx \widetilde{\mathbf{R}}^{2}$

from disks of radius $\frac{1}{4}$ around each half integer lattice point the bijection is the identity map. Identify $\mathbf{R}^{2}$ with $\mathbf{C}$ in the usual way. Let $D \subset \mathbf{C}$ be the disk of radius $\frac{1}{4}, \tilde{D}:=D \times S^{1} / \sim$ and $A \subset \mathbf{C}$ the disk of radius $\frac{1}{4}$ with an open disk of radius $\frac{1}{8}$ around the origin removed, that is, an annulus. Let $\eta: \mathbf{R} \rightarrow[0,1]$ be a smooth (cutoff) function with

$$
\eta(t)= \begin{cases}0 & t \leq \frac{1}{8} \\ \text { a homeomorphism onto }[0,1] & \frac{1}{8} \leq t \leq \frac{1}{4} \\ 1 & t \geq \frac{1}{4}\end{cases}
$$

Then the bijection $h: A \rightarrow \tilde{D}$ is given by $h(z):=\left(\eta(|z|) \cdot z, \frac{z}{|z|}\right) \in \tilde{D}$. We give $\widetilde{\mathbf{R}}^{2}$ the topology that makes the bijection $h: \dot{\mathbf{R}}^{2} \rightarrow \widetilde{\mathbf{R}}^{2}$ into a homeomorphism. Notice that $h$ is a diffeomorphism away from $\left(\frac{1}{2} \mathbf{Z}\right)^{2}$.

Theorem 3.2.2 Let $(\alpha, \beta) \in \mathbf{R}^{2}$ and $\theta \in S^{1} \subset \mathbf{C}$. Then

(1) $P_{\alpha, \beta}^{ \pm}: \mathbf{R}^{2}-\left(\frac{1}{2} \mathbf{Z}\right)^{2} \rightarrow\left\{\right.$ closed subspaces of $\left.L^{2}\left(\Omega^{0+1+2}(T ; s u(2))\right)\right\}$ is continuous.

(2) Moreover $\lim _{t \rightarrow 0^{+}} P_{(\alpha, \beta) \pm t(\operatorname{Re} \theta, \operatorname{Im} \theta)}^{ \pm}$exists and we can define

$$
K_{(\alpha, \beta, \theta)}^{ \pm}:=\lim _{t \rightarrow 0^{+}} P_{(\alpha, \beta) \pm t(\operatorname{Re} \theta, \operatorname{Im} \theta)}^{ \pm} / P_{(\alpha, \beta)}^{ \pm} \subset \mathcal{H}_{(\alpha, \beta)}^{0+1+2}(T ; \mathbf{C} j) .
$$

Note that $K_{(\alpha, \beta, \theta)}^{ \pm}=0$ for $(\alpha, \beta) \notin\left(\frac{1}{2} \mathbf{Z}\right)^{2}$.

(3) $P_{\alpha, \beta}^{ \pm} \oplus K_{(\alpha, \beta, \theta)}^{ \pm}: \widetilde{\mathbf{R}}^{2} \rightarrow\left\{\right.$ closed subspaces of $\left.L^{2}\left(\Omega^{0+1+2}(T ; s u(2))\right)\right\}$ is continuous.

(4) If $(\alpha, \beta)=\frac{1}{2}(r, s) \in\left(\frac{1}{2} \mathbf{Z}\right)^{2}$, then $K_{(\alpha, \beta, \theta)}^{ \pm}=\left\langle\left\{\psi_{1}^{ \pm} j, \psi_{2}^{ \pm} j, \psi_{1}^{ \pm} k, \psi_{2}^{ \pm} k\right\}\right\rangle$

where $\quad \psi_{1}^{ \pm}=e^{i(r m+s l)}(1 \mp(i \operatorname{Im} \theta d m-i \operatorname{Re} \theta d l))$ 
and

$$
\psi_{2}^{ \pm}=e^{i(r m+s l)}(d m \wedge d l \pm(i \operatorname{Re} \theta d m+i \operatorname{Im} \theta d l))
$$

Notice from the explicit description of $K^{ \pm}$that $K_{(\alpha, \beta, \theta)}^{ \pm}=K_{(\alpha, \beta,-\theta)}^{\mp}$. Before we prove this theorem, an eigenspace decomposition will be useful to compute $K_{(\alpha, \beta, \theta)}^{ \pm}$explicitly and study the behaviour of the family $P_{(\alpha, \beta)}^{ \pm} \oplus K_{(\alpha, \beta, \theta)}^{ \pm}$around the half integer lattice. It is a lengthy but straightforward computation, which we will leave to the reader.

Proposition 3.2.3 Fix $a=a_{\alpha, \beta}$. We have the orthogonal decomposition

$$
L^{2}\left(\Omega^{0+1+2}(T ; s u(2))\right)=\bigoplus_{(r, s) \in \mathbf{Z}^{2}} E_{r, s} L^{2} \oplus \bigoplus_{(r, s) \in \mathbf{Z}^{2}} E_{r, s}^{\prime} L^{2}
$$

where forms in

$$
E_{r, s}=\left\{f \sin (r m+s l)+g \cos (r m+s l) \mid f, g \in \mathcal{H}^{0+1+2}(T ; \mathbf{R} i)\right\}
$$

are eigenvectors of $\Delta_{a}$ with eigenvalue $r^{2}+s^{2}$, and forms in

$$
E_{r, s}^{\prime}=\left\{e^{i(r m+s l)} f \mid f \in \mathcal{H}^{0+1+2}(T ; \mathbf{C} j)\right\}
$$

are eigenvectors of $\Delta_{a}$ with eigenvalue $(r-2 \alpha)^{2}+(s-2 \beta)^{2} \cdot \mathcal{H}^{0+1+2}(T ; \mathbf{R} i)$ and $\mathcal{H}^{0+1+2}(T ; \mathbf{C} j)$ are the harmonic forms of the untwisted Laplacian.

Proof of Theorem 3.2.2 For the continuity of $P_{\alpha, \beta}^{ \pm}$away from $\left(\frac{1}{2} \mathbf{Z}\right)^{2}$ see 21]. We show that $\lim _{t \rightarrow 0^{+}} P_{(\alpha, \beta) \pm t(\operatorname{Re} \theta, \operatorname{Im} \theta)}^{ \pm}$exists by explicitly computing $K_{(\alpha, \beta, \theta)}^{ \pm}$for $(\alpha, \beta)=\frac{1}{2}(r, s) \in\left(\frac{1}{2} \mathbf{Z}\right)^{2}$.

Let $\left(\alpha_{t}, \beta_{t}\right)=\frac{1}{2}(r, s)+\frac{1}{2} t(\operatorname{Re} \theta, \operatorname{Im} \theta), t>0$. By Proposition $3.2 .3 \varphi=e^{i(r m+s l)} j$ is an eigenvector of $\Delta_{\left(\alpha_{t}, \beta_{t}\right)}=\Delta_{a_{\left(\alpha_{t}, \beta_{t}\right)}}$ with eigenvalue $t^{2}=\left(r-2 \alpha_{t}\right)^{2}+(s-$ $\left.2 \beta_{t}\right)^{2}$. Then by Lemma 2.5.1

$$
\varphi \pm \frac{1}{t} S_{\left(\alpha_{t}, \beta_{t}\right)} \varphi=e^{i(r m+s l)}\left(1 \mp \frac{1}{t} i\left(r-2 \alpha_{t}\right) d l \pm \frac{1}{t} i\left(s-2 \beta_{t}\right) d m\right) j
$$

is a $\pm t$-eigenvector of $S_{\left(\alpha_{t}, \beta_{t}\right)}$. This yields

$$
\lim _{t \rightarrow 0}\left(\varphi \pm \frac{1}{t} S_{\left(\alpha_{t}, \beta_{t}\right)} \varphi\right)=e^{i(r m+s l)}(1 \mp(i \operatorname{Im} \theta d m-i \operatorname{Re} \theta d l)) .
$$

For $\varphi=e^{i(r m+s l)} j d m \wedge d l$ we similarly get

$$
\lim _{t \rightarrow 0}\left(\varphi \pm \frac{1}{t} S_{\left(\alpha_{t}, \beta_{t}\right)} \varphi\right)=e^{i(r m+s l)}(d m \wedge d l \pm(i \operatorname{Re} \theta d m+i \operatorname{Im} \theta d l)) j .
$$

Repeating the same computation for $\varphi=e^{i(r m+s l)} k$ and $\varphi=e^{i(r m+s l)} k d m \wedge d l$ yields a total of 8 linearly independent eigenvectors, which lie in either $K_{(\alpha, \beta, \theta)}^{+}$ 
or $K_{(\alpha, \beta, \theta)}^{-}$. By Proposition 3.1.2 the $\mathbf{C} j$ part of $\operatorname{Ker} S_{(\alpha, \beta, \theta)}$ is 8-dimensional. Thus the $L^{2}$-span of the above eigenvectors make up $K_{(\alpha, \beta, \theta)}^{+}$or $K_{(\alpha, \beta, \theta)}^{-}$. This completes the computation of $K_{(\alpha, \beta, \theta)}^{ \pm}$and shows that $\lim _{t \rightarrow 0^{+}} P_{(\alpha, \beta) \pm t(\operatorname{Re} \theta, \operatorname{Im} \theta)}^{ \pm}$ exists.

We are left with proving the continuity of $P_{\alpha, \beta}^{+} \oplus K_{\alpha, \beta, \theta}^{+}$parametrized by $(\widetilde{\mathbf{R}})^{2}$. Away from $\left(\frac{1}{2} \mathbf{Z}\right)^{2}$ the family $P_{\alpha, \beta}^{+} \oplus K_{\alpha, \beta, \theta}^{+}$is continuous since $K_{\alpha, \beta, \theta}^{+}=0$. To show continuity at half integer lattice points, it suffices to show that for any continuous path $\tilde{\varrho}_{t}=\left(\tilde{\alpha}_{t}, \tilde{\beta}_{t}, \theta_{t}\right)$ in $\widetilde{\mathbf{R}}^{2}$ that limits to $\tilde{\varrho}(0)=\left(0,0, \theta_{0}\right)$ we have

$$
\lim _{t \rightarrow 0} P_{\tilde{\alpha}_{t}, \tilde{\beta}_{t}}^{+} \oplus K_{\tilde{\varrho}_{t}}^{+}=P_{0,0}^{+} \oplus K_{0,0, \theta}^{+} \text {. }
$$

For all other half integer lattice points the argument is the same. By definition $\tilde{\varrho}_{t}$ is continuous in a ball of radius $\frac{1}{8}$ around $(0,0)$, if $h^{-1} \circ \varrho_{t}=\varrho_{t}=\left(\alpha_{t}, \beta_{t}\right)$ is continuous in $\mathbf{R}^{2}$, where $h: \dot{\mathbf{R}}^{2} \rightarrow \widetilde{\mathbf{R}}^{2}$ is the homeomorphism given in Definition 3.2.1 We consider two cases:

(1) If $\left|\varrho_{t}\right|=\frac{1}{8}$ for $t$ small, then $\tilde{\varrho}_{t}=\left(0,0, \theta_{t}\right)$ and $\theta_{t}$ continuous for small $t$. Elementary triangle equality arguments applied to $P_{(0,0)+s(\operatorname{Re} \theta, \operatorname{Im} \theta)}^{+}, s$ small, show that $K_{0,0, \theta_{t}}^{+}$is continuous.

(2) Let $\left|\varrho_{t}\right| \neq \frac{1}{8}$ for $t>0$ small. We have $\left(\tilde{\alpha}_{t}, \tilde{\beta}_{t}\right)=\left(\alpha_{t}, \beta_{t}\right) \eta\left(\sqrt{\alpha_{t}^{2}+\beta_{t}^{2}}\right)$ and $\theta_{0}=8\left(\alpha_{0}+i \beta_{0}\right) \in S^{1}$. To check that $K_{0,0, \theta_{0}}^{+}=\lim _{t \rightarrow 0}\left(P_{\tilde{\alpha}_{t}, \tilde{\beta}_{t}}^{+} / P_{0,0}^{+}\right)$, observe that by Lemma 2.5.1 the $\left(\left(2 \tilde{\alpha}_{t}\right)^{2}+\left(2 \tilde{\beta}_{t}\right)^{2}\right)$-eigenvector $\varphi=j$ of $\Delta_{\tilde{\alpha}_{t}, \tilde{\beta}_{t}}$ yields a $2 \sqrt{\tilde{\alpha}_{t}^{2}+\tilde{\beta}_{t}^{2}}$-eigenvector of $S_{\tilde{\alpha}_{t}, \tilde{\beta}_{t}}$ :

$$
\begin{aligned}
& \varphi+\frac{1}{2 \sqrt{\tilde{\alpha}_{t}^{2}+\tilde{\beta}_{t}^{2}}} S_{\tilde{\alpha}_{t}, \tilde{\beta}_{t}} \varphi=\varphi+\frac{2 i \tilde{\alpha}_{t} d l-2 i \tilde{\beta}_{t} d m}{2 \sqrt{\tilde{\alpha}_{t}^{2}+\tilde{\beta}_{t}^{2}}} \\
&=j+\frac{i \alpha_{t} \eta\left(\sqrt{\alpha_{t}^{2}+\beta_{t}^{2}}\right) d l-i \beta_{t} \eta\left(\sqrt{\alpha_{t}^{2}+\beta_{t}^{2}}\right) d m}{\sqrt{\alpha_{t}^{2} \eta\left(\sqrt{\alpha_{t}^{2}+\beta_{t}^{2}}\right)^{2}+\beta_{t}^{2} \eta\left(\sqrt{\alpha_{t}^{2}+\beta_{t}^{2}}\right)^{2}}} j \\
&=j+\frac{i \alpha_{t} d l-i \beta_{t} d m}{\sqrt{\alpha_{t}^{2}+\beta_{t}^{2}}} j \\
& \stackrel{t \rightarrow 0}{\longrightarrow} \quad j+8\left(i \alpha_{0} d l-i \beta_{0} d m\right) j=j-(i \operatorname{Im} \theta d m-i \operatorname{Re} \theta d l) j
\end{aligned}
$$

The same computation for the other $2 \sqrt{\tilde{\alpha}_{t}^{2}+\tilde{\beta}_{t}^{2}}$-eigenvectors of $S_{\tilde{\alpha}_{t}, \tilde{\beta}_{t}}$ yields the other basis elements of $K_{0,0, \theta}^{+}$as given in the statement of Theorem 3.2 .2

Thus $P_{\alpha, \beta}^{+} \oplus K_{\alpha, \beta, \theta}^{+}$is a continuous family parametrized by $(\widetilde{\mathbf{R}})^{2}$. 
Definition 3.2.4 For a continuous family of Lagrangians $L$ of $\mathcal{H}^{0+1+2}(T ; \mathbf{R} i)$ parametrized by a subset $U \subset \widetilde{\mathbf{R}}^{2}$, define a family $\mathcal{P}^{ \pm}(L)$ of subspaces of $L^{2}\left(\Omega^{0+1+2}(T ; s u(2))\right)$ parametrized by $U$ as follows

$$
\mathcal{P}_{(\alpha, \beta, \theta)}^{ \pm}(L):=\left(P_{\mathbf{R} i}^{ \pm} \oplus L_{(\alpha, \beta, \theta)}\right) \oplus\left(P_{\mathbf{C} j,(\alpha, \beta)}^{ \pm} \oplus K_{(\alpha, \beta, \theta)}^{ \pm}\right)
$$

For our application, the family of Lagrangians $L_{\alpha, \beta, \theta}$ will be independent of $(\alpha, \beta, \theta)$. Notice that $K_{(\alpha, \beta, \theta)}$ vanishes away from $\left(\frac{1}{2} \mathbf{Z}\right)^{2}$, while we have "blown up" the points of $\left(\frac{1}{2} \mathbf{Z}\right)^{2}$ and removed the singularities that paths through $\left(\frac{1}{2} \mathbf{Z}\right)^{2}$ encounter. Here is a corollary of Theorem 3.2.2 which is important for the following sections.

Theorem 3.2.5 $\mathcal{P}^{ \pm}(L)$ is a continuous family of Lagrangians parametrized by $\widetilde{\mathbf{R}}^{2}$.

\section{Spectral flow on a closed 3-manifold}

In this section we develop a splitting formula for spectral flow, which expresses spectral flow of the odd signature operator between flat connections on a closed 3 -manifold $M$ in terms of spectral flow on the solid torus $S$ and its complement $X$ with the Atiyah-Patodi-Singer boundary conditions from Section 3 Even though Nicolaescu 24] and Daniel [10] provide us by way of Theorem 2.7.1] with an elegant expression of spectral flow in terms of the Maslov index of the respective Cauchy data spaces, it is not immediately applicable to spectral flow computations, since the Cauchy data spaces themselves are complicated objects. The purpose of the splitting formula in Theorem 4.3.1, however, is to make computations of spectral flow easier by shifting the problem to two more tractable ones. In fact, an explicit way to compute the spectral flow on $S$ is given in Theorem 5.3.3. One only needs to do some work computing the spectral flow on $X$. The main application to keep in mind is the spectral flow computation of the twisted odd signature operator between flat connections, whenever it is possible to find a path between them which is flat on $X$, because then we can use topology to try computing the spectral flow on $X$.

\section{$4.1 \quad$ Objective}

The setup in this section is as follows. Let $M=X \cup_{T} S$ be a closed 3-manifold with $S$ being the solid torus and $T$ the torus as in Section 2.1. Let $A_{t}$ be a path of $S U(2)$ connections on $M$ with the following properties: 
(1) $A_{t}$ is in cylindrical form and flat in a collar of $T$.

(2) $A_{t}$ restricts to the path $a_{\varrho(t)}$ (see Definition 3.1.1) on $T$ for some path $\varrho$ in $\widetilde{\mathbf{R}}^{2}$ with $\pi \circ \varrho=\varrho$, where $\pi: \widetilde{\mathbf{R}}^{2} \rightarrow \mathbf{R}^{2}$ is the projection onto the $\mathbf{R}^{2}$-factor.

(3) $A_{0}$ and $A_{1}$ are flat on $M$.

The goal in this section is to find a splitting formula expressing $\operatorname{SF}\left(D_{A_{t}}\right)$ in terms of spectral flow on $X$ and $S$. Notice that, while the spectral flow on $X$ and $S$ depends on the lift $\tilde{\rho}$ of $\rho$ in property (2), $A_{t}$ and $\operatorname{SF}\left(D_{A_{t}}\right)$ are independent of it.

The above assumptions do not limit the applicability of the splitting formula in Theorem 4.3.1. Indeed, the spectral flow of the odd signature operator along a path of $S U(2)$ connections $A_{t}$ with flat endpoints depends only on $A_{0}$ and $A_{1}$, which we can gauge transform by some $g_{\varepsilon}, \varepsilon=0,1$ so that $\left.g_{\varepsilon} \cdot A_{\varepsilon}\right|_{N(T)}=a_{\alpha_{\varepsilon}, \beta_{\varepsilon}}$. The change in spectral flow is given by Proposition 2.4.1. We can extend the path $(1-t) a_{\alpha_{0}, \beta_{0}}+t a_{\alpha_{1}, \beta_{1}}$ by obstruction theory to a path of connections on $M$ with endpoints $\left.g_{0} \cdot A_{0}\right|_{N(T)}$ and $\left.g_{1} \cdot A_{1}\right|_{N(T)}$, since $[0,1] \times \Omega^{*}(M ; s u(2))$ is contractible.

\subsection{The scattering Lagrangian of $D_{A}$ on $S$}

Before we start with the discussion of the spectral flow on $M$, we analyze the scattering Lagrangian of $D_{A}$ at a flat connection $A$ on $S$ which restricts to $a_{\alpha, \beta}=-i \alpha d m-i \beta d l$ on $T$, because its explicit description plays a central role in the splitting theorem.

The scattering Lagrangian of $D_{A}$ for $A=-i \beta d l$ can be computed directly.

Consider a flat $S U(2)$ connection $A$ on $S$ with $\left.A\right|_{T}=a_{\alpha, \beta}$. One observes that $p: \pi_{1}(T) \rightarrow \pi_{1}(S)=\left\langle\pi_{1}(T) \mid \mu=1\right\rangle$ is a surjection and $\operatorname{hol}(A) \circ p=\operatorname{hol}\left(a_{\alpha, \beta}\right)$. Thus we have $\operatorname{hol}\left(a_{\alpha, \beta}\right)(\mu)=1$, that is, $\alpha \in \mathbf{Z}$, and in view of Proposition 2.6.1 the scattering Lagrangian depends only on $a_{\alpha, \beta}$. Therefore we can use a gauge transformation $g$ with $\left.g\right|_{T}=e^{i \alpha m}$ on the boundary to compute $\mathscr{L}_{S, g \cdot A}=$ $\operatorname{ad}_{g} \mathscr{L}_{S, A}$. This yields the following.

Lemma 4.2.1 Suppose $A$ is a flat $S U(2)$ connection on $S$ with $\left.A\right|_{T}=a_{\alpha, \beta}$. Then $\alpha \in \mathbf{Z}$ and

- $\mathscr{L}_{S, A}=\mathbf{R} i \oplus \mathbf{R} i d l$ for $\beta \in \mathbf{R}-\frac{1}{2} \mathbf{Z}$, 
- $\mathscr{L}_{S, A}=\hat{\mathscr{L}}_{S, A} \oplus \check{\mathscr{L}}_{S, A}$ for $\beta \in \frac{1}{2} \mathbf{Z}$, where

$$
\begin{aligned}
\hat{\mathscr{L}}_{S, A} & =\mathbf{R} i \oplus \mathbf{R} i d l \\
\check{\mathscr{L}}_{S, A} & =e^{i(2 \alpha m+2 \beta l)}(\mathbf{C} j \oplus \mathbf{C} j d l)
\end{aligned}
$$

Thus we make the following definition.

Definition 4.2.2 For $(\alpha, \beta) \in \mathbf{R}^{2}$ define $\hat{\mathscr{L}}_{S, A_{(\alpha, \beta)}}:=\mathbf{R} i \oplus \mathbf{R} i d l$. Note that $\hat{\mathscr{L}}_{S, A_{(\alpha, \beta)}}=\mathcal{H}^{0}(T ; \mathbf{R} i) \oplus \mathbf{R} i d l \subset \mathcal{H}^{0+1+2}(T ; \mathbf{R} i)$.

\subsection{A splitting formula for spectral flow}

We will derive the following splitting formula.

Theorem 4.3.1 Let $M=X \cup_{T} S$ be a closed 3-manifold with $S$ being the solid torus and $T$ the torus as in Section [2.1. Let $A_{t}$ be a path of $S U(2)$ connections on $M$ with the following properties:

(1) $A_{t}$ is in cylindrical form and flat in a collar of $T$.

(2) $A_{t}$ restricts to the path $a_{\varrho(t)}$ on $T$ for some path $\tilde{\varrho}$ in $\widetilde{\mathbf{R}}^{2}$ with $\pi \circ \varrho=\varrho$, where $\pi: \widetilde{\mathbf{R}}^{2} \rightarrow \mathbf{R}^{2}$ is the projection onto the $\mathbf{R}^{2}$-factor.

(3) $A_{0}$ and $A_{1}$ are flat on $M$.

Then we have the splitting formula:

$$
\begin{aligned}
\operatorname{SF}\left(D_{A_{t}}\right)= & \left.\left.\operatorname{SF}\left(\left.D_{A_{t}}\right|_{S} ; \mathcal{P}_{\tilde{\varrho}(t)}^{+}\left(J \hat{\mathscr{L}}_{S}\right)\right)\right)+\operatorname{SF}\left(\left.D_{A_{t}}\right|_{X} ; \mathcal{P}_{\tilde{\varrho}(t)}^{-}\left(\hat{\mathscr{L}}_{S}\right)\right)\right) \\
& +\tau_{\mu}\left(J \mathscr{L}_{S, 0}, K_{\varrho_{0}, i}^{+} \oplus J \hat{\mathscr{L}}_{S, 0}, \mathscr{L}_{X, 0}\right)-\tau_{\mu}\left(J \mathscr{L}_{S, 1}, K_{\varrho_{1}, i}^{+} \oplus J \hat{\mathscr{L}}_{S, 1}, \mathscr{L}_{X, 1}\right) .
\end{aligned}
$$

The proof of Theorem 4.3.1 is deferred to the next section. However, the idea is the following. First we relate the spectral flow to the Maslov index of the Cauchy data spaces using Nicolaescu's splitting theorem. Next we use homotopy invariance of the Maslov index and Nicolaescu's adiabatic limit theorem to find homotopic paths of Lagrangians, parts of which correspond to a Maslov index of Cauchy data space with APS boundary conditions. Now we can use additivity of pairs of paths of Lagrangians and apply the relative version of Nicolaescu's splitting theorem to two of the parts, which yields the first two summands of the formula. The remaining summands will either vanish or simplify to the two Maslov triple indices involving $K_{\alpha, \beta, \theta}^{ \pm}$from Theorem 3.2.2 the scattering Lagrangians $\mathscr{L}_{S, \varepsilon}=\mathscr{L}_{S, A_{\varepsilon}}$ and $\mathscr{L}_{X, \varepsilon}=\mathscr{L}_{X, A_{\varepsilon}}$ at $\varepsilon=0,1$, as well as $\hat{\mathscr{L}}_{S, \varepsilon}=$ $\hat{\mathscr{L}}_{S, A_{\varepsilon}}$ from Lemma 4.2.1

The situation is particularly nice and straightforward when 
(1) $D_{A_{0}}$ and $D_{A_{1}}$ restricted to $X$ have non-resonance level 0 , and

(2) $\varrho(t) \notin\left(\frac{1}{2} \mathbf{Z}\right)^{2}$ for all $t \in[0,1]$, in which case $K_{(\varrho(t), \theta)}^{ \pm}=0$.

We describe the derivation of our splitting formula without these assumptions.

Fix the path $R_{t}:=\frac{t}{1-t}$ which corresponds to the parameter that stretches the collar from 0 to $\infty$. Stretching the collar of $S$ yields the adiabatic limit $\Lambda_{S, \varepsilon}^{R_{1}}=P_{S, \varepsilon}^{-} \oplus \mathscr{L}_{S, \varepsilon}$, because $\left.D_{A_{\varepsilon}}\right|_{S}$ has non-resonance level 0 when $\varepsilon=0,1$. Theorem 2.6.4 describes $\Lambda_{X, \varepsilon}^{R_{1}}$.

Since $K_{\alpha, \beta, \theta}^{ \pm} \neq K_{\alpha, \beta,-\theta}^{ \pm}$for $(\alpha, \beta) \in\left(\frac{1}{2} \mathbf{Z}\right)^{2}$ (see Theorem 3.2.2), we will use the APS boundary conditions $\mathcal{P}_{\tilde{\varrho}(t)}^{-}\left(\hat{\mathscr{L}}_{S}\right)$ and $\mathcal{P}_{\tilde{\rho}(t)}^{+}\left(J \mathscr{L}_{S}\right)$ for our splitting formula with the notation introduced in Definition 3.2.4 and Lemma 4.2.1, which were shown to be continuous for a continuous lift $\varrho$ of the path $\varrho$ from $\mathbf{R}^{2}$ to $\widetilde{\mathbf{R}}^{2}$.

We will need to "rotate" the adiabatic limit of $\left.D_{A_{0}}\right|_{S}$ and $\left.D_{A_{1}}\right|_{X}$ to our preferred APS boundary conditions.

Definition 4.3.2 We introduce the paths $P_{1, \nu}^{+} \oplus L_{W, t} \oplus d_{a_{1}}\left(E_{1, \nu}^{-}\right) \oplus L_{X, t}$ for $X$ and $P_{0}^{-} \oplus L_{S, t}$ for $S$ as follows:

(1) Let $L_{X, t}$ be a path of Lagrangians in $H^{0+1+2}(T ; s u(2))$ from the scattering Lagrangian $\mathscr{L}_{X, 1}$ to $J \hat{\mathscr{L}}_{S, 1} \oplus K_{\varrho_{1}, i}^{+}$.

(2) $\quad L_{W, t}=e^{J \pi t} W_{a_{1}} \oplus J\left(W_{a_{1}}^{\perp}\right)$ is a path of Lagrangians in $d_{a_{t}}\left(E_{a_{1}, \nu}^{+}\right) \oplus d^{*}\left(E_{a_{1}, \nu}^{-}\right)$ from $W_{a_{1}} \oplus J\left(W_{a_{1}}^{\perp}\right)$ to $d^{*}\left(E_{a_{1}, \nu}^{-}\right)=J\left(W_{\alpha}\right) \oplus J\left(W_{a}^{\perp}\right) \subset P_{1}^{+}$.

(3) For $\varepsilon=0,1$ let $L_{S, \varepsilon, t}=\hat{\mathscr{L}}_{S, \varepsilon} \oplus \check{L}_{S, \varepsilon, t}$ be a path of Lagrangians in $H^{0+1+2}(T ; s u(2))$, where $\check{L}_{S, \varepsilon, t}$ is an arbitrary path from $\check{\mathscr{L}}_{S, \varepsilon}$ to $K_{\varrho_{\varepsilon}, i}^{-}$. As in Lemma 4.2.1 $\check{\mathscr{L}}_{S, \varepsilon}$ denotes the $\mathbf{C} j$ part of $\mathscr{L}_{S, \varepsilon}$. Set $L_{S, t}=L_{S, 0, t}$.

Then it is straightforward to check that the composition of paths $\left(\mathscr{M}_{i}(t), \mathscr{N}_{i}(t)\right)$ given in Table 1 is homotopic to the path of pairs of Cauchy data spaces $\left(\Lambda_{S, t}, \Lambda_{X, t}\right)$. The table shows the $i$-th paths $\mathscr{M}_{i}$ and $\mathscr{N}_{i}$ in the second and fifth column. The third and fourth column give the endpoints of $\mathscr{M}_{i}$ and $\mathscr{N}_{i}$ as a reference.

If $\varrho_{\varepsilon} \notin\left(\frac{1}{2} \mathbf{Z}\right)^{2}$ for $\varepsilon=0,1$, then we have $K_{\varrho_{\varepsilon}, i}^{ \pm}=0$, thus

$$
\tau_{\mu}\left(J \mathscr{L}_{S, 0}, K_{\varrho_{0}, i}^{+} \oplus J \hat{\mathscr{L}}_{S, 0}, \mathscr{L}_{X, 0}\right)=\tau_{\mu}\left(J \mathscr{L}_{S, 1}, K_{\varrho_{1}, i}^{+} \oplus J \hat{\mathscr{L}}_{S, 1}, \mathscr{L}_{X, 1}\right)=0 .
$$

This yields the following. 


\begin{tabular}{|c|c|c|c|c|}
\hline$i$ & paths $\mathscr{M}_{i}(t)$ & Endpoints & of $\mathscr{M}_{i}$ and $\mathscr{N}_{i}$ & paths $\mathscr{N}_{i}(t)$ \\
\hline \multirow[b]{2}{*}{1} & & \multirow{2}{*}{$\Lambda_{S, 0}$} & \multirow{2}{*}{$\Lambda_{X, 0}$} & \multirow{2}{*}{$\Lambda_{X, 0}^{R_{t}}$} \\
\hline & $\Lambda_{S, 0}^{R_{t}}$ & & & \\
\hline \multirow{2}{*}{2} & \multirow{2}{*}{$P_{0}^{-} \oplus L_{S, t}$} & $P_{0}^{-} \oplus \mathscr{L}_{S, 0}$ & $\Lambda_{X, 0}^{\infty}$ & \multirow{2}{*}{$\Lambda_{X, 0}^{R_{1-t}}$} \\
\hline & & \multirow{2}{*}{$\mathcal{P}_{\tilde{\varrho}(0)}^{-}\left(\hat{\mathscr{L}}_{S}\right)$} & \multirow{2}{*}{$\Lambda_{X, 0}$} & \\
\hline 3 & $\mathcal{P}_{\tilde{\varrho}(t)}^{-}\left(\hat{\mathscr{L}}_{S}\right)$ & & & $\Lambda_{X, t}$ \\
\hline \multirow{2}{*}{4} & conctant & $\mathcal{P}_{\tilde{\varrho}(1)}^{-}\left(\hat{\mathscr{L}}_{S}\right)$ & $\Lambda_{X, 1}$ & \multirow{2}{*}{$\Lambda_{X, 1}^{R_{t}}$} \\
\hline & constant & \multirow{2}{*}{$\mathcal{P}_{\tilde{\varrho}(1)}^{-}\left(\hat{\mathscr{L}}_{S}\right)$} & \multirow{2}{*}{$\Lambda_{X, 1}^{\infty}$} & \\
\hline 5 & constant & & & $P_{a_{1}, \nu}^{+} \oplus L_{W, t} \oplus d_{a_{1}}\left(E_{a_{1}, \nu}^{-}\right) \oplus L_{X, t}$ \\
\hline \multirow{2}{*}{6} & \multirow{2}{*}{$\mathcal{P}_{\tilde{\varrho}(1-t)}^{-}\left(\hat{\mathscr{L}}_{S}\right)$} & \multirow{2}{*}{$\mathcal{P}_{\tilde{\varrho}(1)}^{-}\left(\hat{\mathscr{L}}_{S}\right)$} & $\mathcal{P}_{\tilde{\varrho}(1)}^{+}\left(J \hat{\mathscr{L}}_{S}\right)$ & \multirow{2}{*}{$\mathcal{P}_{\tilde{\varrho}(1-t)}^{+}\left(J \hat{\mathscr{L}}_{S}\right)$} \\
\hline & & & \multirow{2}{*}{$\mathcal{P}_{\tilde{\varrho}(0)}^{+}\left(J \hat{\mathscr{L}}_{S}\right)$} & \\
\hline 7 & $P_{0}^{-} \oplus L_{S, 1-t}$ & $\mathcal{P}_{\tilde{\varrho}(0)}^{-}\left(\hat{\mathscr{L}}_{S}\right)$ & & \multirow[t]{2}{*}{ constant } \\
\hline & \multirow{2}{*}{$\Lambda_{S, 0}^{R_{1-t}}$} & $P_{0}^{-} \oplus \mathscr{L}_{S, 0}$ & $\mathcal{P}_{\tilde{\varrho}(0)}^{+}\left(J \hat{\mathscr{L}}_{S}\right)$ & \\
\hline 8 & & $\Delta_{\mathrm{c}}$ & $\mathcal{P}^{+}\left(I \hat{\mathscr{\rho}}_{c}\right)$ & constant \\
\hline 9 & $\Lambda_{S, t}$ & & $\tilde{\varrho}(0)(J \propto S)$ & $\mathcal{P}_{\tilde{\varrho}(t)}^{+}\left(J \hat{\mathscr{L}}_{S}\right)$ \\
\hline 10 & $\Lambda_{t}^{R_{t}}$ & $\Lambda_{S, 1}$ & $\mathcal{P}_{\tilde{\varrho}(1)}^{+}\left(J \mathscr{L}_{S}\right)$ & $\left.P^{+} \oplus L_{\mathrm{W} 1}, \oplus d \mathrm{~d}^{-} \quad F^{-}\right) \oplus L_{\mathrm{Y}}{ }^{-}$ \\
\hline 10 & ${ }^{\Lambda_{S, 1}}$ & $\Delta_{c 1}^{\infty}$ & $\Delta_{Y}^{\infty}$ & $P_{a_{1}, \nu} \oplus L_{W, 1-t} \oplus a_{a_{1}\left(L_{a_{1}, \nu}\right)} \oplus L_{X, 1-t}$ \\
\hline 11 & $\Lambda_{S, 1}^{R_{1-t}}$ & ${ }_{S, 1}$ & ${ }_{X, 1}$ & $\Lambda_{X, 1}^{R_{1-t}}$ \\
\hline & & $\Lambda_{S, 1}$ & $\Lambda_{X, 1}$ & \\
\hline
\end{tabular}

Table 1: The paths homotopic to $\Lambda_{S, t}$ and $\Lambda_{X, t}$ broken up into pieces

Corollary 4.3.3 We make the same assumptions as in Theorem 4.3.1. Then, if $\varrho_{\varepsilon} \notin\left(\frac{1}{2} \mathbf{Z}\right)^{2}$ for $\varepsilon=0,1$ we get:

$$
\mathrm{SF}\left(D_{A_{t}}\right)=\operatorname{SF}\left(\left.D_{A_{t}}\right|_{S} ; \mathcal{P}_{\tilde{\varrho}(t)}^{+}\left(J \hat{\mathscr{L}}_{S}\right)\right)+\operatorname{SF}\left(\left.D_{A_{t}}\right|_{X} ; \mathcal{P}_{\tilde{\varrho}(t)}^{-}\left(\hat{\mathscr{L}}_{S}\right)\right)
$$

A way to compute $\operatorname{SF}\left(\left.D_{A_{t}}\right|_{S} ; \mathcal{P}_{\tilde{\varrho}(t)}^{+}\left(J \hat{\mathscr{L}}_{S}\right)\right)$ will be given in Theorem 5.3.3. We will compute $\operatorname{SF}\left(\left.D_{A_{t}}\right|_{X} ; \mathcal{P}_{\tilde{\varrho}(t)}^{-}\left(\hat{\mathscr{L}}_{S}\right)\right)$ for our main application in Section 6 , where $M$ is a torus-bundle over $S^{1}$ and $A_{t}$ is flat on $X$. 


\subsection{Proof of Theorem 4.3.1}

By Lemma 2.6.5 the Maslov indices $\operatorname{Mas}\left(\mathscr{M}_{i}, \mathscr{N}_{i}\right)$ vanish for $i=1,4,8,11$. By Theorem 2.7.2 we have

$$
\operatorname{Mas}\left(\mathscr{M}_{9}, \mathscr{N}_{9}\right)=\operatorname{SF}\left(\left.D_{A_{t}}\right|_{S} ; \mathcal{P}_{\tilde{\varrho}(t)}^{+}\left(J \hat{\mathscr{L}}_{S}\right)\right) .
$$

Since $\mathcal{P}^{-}\left(\hat{\mathscr{L}}_{S, t}\right)=\left(\mathcal{P}_{\tilde{\varrho}(t)}^{+}\left(J \hat{\mathscr{L}}_{S}\right)\right)^{\perp}$ for all $t$, the Maslov index $\operatorname{Mas}\left(\mathscr{M}_{6}, \mathscr{N}_{6}\right)$ vanishes. Again by Theorem 2.7 .2 we have

$$
\operatorname{Mas}\left(\mathscr{M}_{3}, \mathscr{N}_{3}\right)=\operatorname{SF}\left(\left.D_{A_{t}}\right|_{X} ; \mathcal{P}^{-}\left(\hat{\mathscr{L}}_{S}\right)\right)
$$

Let us focus our attention on the rest of the paths, namely paths 2, 5, 7 and 10.

We may homotop $\mathscr{M}_{2}$ to the composition of the two paths

$$
\begin{aligned}
\mathscr{M}_{2 a} & =P_{0}^{-} \oplus L_{S, 0, t} \\
\mathscr{M}_{2 b} & =P_{0}^{-} \oplus K_{\varrho_{0}, i}^{+} \oplus \hat{\mathscr{L}}_{0} .
\end{aligned}
$$

and $\mathscr{N}_{2}$ to the composition of the two paths

$$
\begin{aligned}
\mathscr{N}_{2 a} & =P_{0, \nu}^{+} \oplus\left(W_{0} \oplus J\left(W_{0}^{\perp}\right)\right) \oplus d^{*}\left(E_{0, \nu}^{-}\right) \oplus \mathscr{L}_{X, 0} \\
\mathscr{N}_{2 b} & =\Lambda_{X, 0}^{R_{1-t}} .
\end{aligned}
$$

By Lemma 2.6.5 $\operatorname{Mas}\left(\mathscr{M}_{2 b}, \mathscr{N}_{2 b}\right)=0$. Then by our choice of $L_{S, 0, t}$ we get

$$
\operatorname{Mas}\left(\mathscr{M}_{2}, \mathscr{N}_{2}\right)=\operatorname{Mas}\left(\mathscr{M}_{2 a}, \mathscr{N}_{2 a}\right)=\operatorname{Mas}\left(L_{S, 0, t}, \mathscr{L}_{X, 0}\right) .
$$

We can directly check:

$$
\left.\operatorname{Mas}\left(\mathscr{M}_{7}, \mathscr{N}_{7}\right)=-\operatorname{Mas}\left(L_{S, 0, t}, K_{\varrho_{0}, i}^{+} \oplus J \hat{\mathscr{L}}_{S, 0}\right)\right) .
$$

Then Lemma 2.7.3 and linearity under the direct sum of triples yield

$$
\begin{aligned}
& \operatorname{Mas}\left(\mathscr{M}_{2}, \mathscr{M}_{2}\right)+\operatorname{Mas}\left(\mathscr{M}_{7}, \mathscr{N}_{7}\right) \\
& =\tau_{\mu}\left(J\left(K_{\varrho_{0}, i}^{-} \oplus \hat{\mathscr{L}}_{S, 0}\right), \mathscr{L}_{X, 0}, K_{\varrho_{0}, i}^{+} \oplus J \hat{\mathscr{L}}_{S, 0}\right)-\tau_{\mu}\left(J \mathscr{L}_{S, 0}, \mathscr{L}_{X, 0}, K_{\varrho_{0}, i}^{+} \oplus J \hat{\mathscr{L}}_{S, 0}\right) \\
& =\operatorname{dim}\left(J \mathscr{L}_{X, 0} \cap\left(K_{\varrho_{0}, i}^{+} \oplus J \hat{\mathscr{L}}_{S, 0}\right)\right)-\tau_{\mu}\left(J \mathscr{L}_{S, 0}, \mathscr{L}_{X, 0}, K_{\varrho_{0}, i}^{+} \oplus J \hat{\mathscr{L}}_{S, 0}\right) \\
& =\tau_{\mu}\left(J \mathscr{L}_{S, 0}, K_{\varrho_{0}, i}^{+} \oplus J \hat{\mathscr{L}}_{S, 0}, \mathscr{L}_{X, 0}\right)
\end{aligned}
$$

We get a similar expression for the paths 5 and 10 . The path $\mathscr{M}_{10}$ can be homotoped to the composition of the two paths

$$
\begin{aligned}
& \mathscr{M}_{10 a}=\Lambda_{S, 1}^{t} \\
& \mathscr{M}_{10 b}=\Lambda_{S, 1}^{\infty}=P_{0}^{-} \oplus \mathscr{L}_{S, 1}
\end{aligned}
$$


and $\mathscr{N}_{10}$ to the composition of the two paths

$$
\begin{aligned}
& \mathscr{N}_{10 a}=\mathcal{P}_{\tilde{\varrho}(1)}^{+}\left(J \hat{\mathscr{L}}_{S}\right) \\
& \mathscr{N}_{10 b}=P_{\nu}^{+} \oplus L_{W, t}
\end{aligned}
$$

Again by Lemma 2.6.5 we get $\operatorname{Mas}\left(\mathscr{M}_{10 a}, \mathscr{N}_{10 a}\right)=0$. Thus

$$
\begin{aligned}
\operatorname{Mas}\left(\mathscr{M}_{10}, \mathscr{N}_{10}\right) & =\operatorname{Mas}\left(P_{1}^{-} \oplus \mathscr{L}_{S, 1}, P_{1, \nu}^{+} \oplus L_{W, 1-t} \oplus d_{a_{1}}\left(E_{1, \nu}^{-}\right) \oplus L_{X, 1-t}\right) \\
& =\operatorname{Mas}\left(E_{1}^{-} \oplus \mathscr{L}_{S, 1}, L_{W, 1-t} \oplus d_{a_{1}}\left(E_{1, \nu}^{-}\right) \oplus L_{X, 1-t}\right) \\
& =\operatorname{Mas}\left(E_{1}^{-}, L_{W, 1-t} \oplus d_{a_{1}}\left(E_{1, \nu}^{-}\right)\right)+\operatorname{Mas}\left(\mathscr{L}_{S, 1}, L_{X, 1-t}\right)
\end{aligned}
$$

while

$$
\begin{aligned}
\operatorname{Mas}\left(\mathscr{M}_{5}, \mathscr{N}_{5}\right) & =\operatorname{Mas}\left(P_{1}^{-} \oplus K_{1, i}^{-} \oplus \hat{\mathscr{L}}_{S, 1}, P_{1, \nu}^{+} \oplus L_{W, t} \oplus d_{a_{1}}\left(E_{1, \nu}^{-}\right) \oplus L_{X, t}\right) \\
& =\operatorname{Mas}\left(E_{1}^{-}, L_{W, t} \oplus d_{a_{1}}\left(E_{1, \nu}^{-}\right)\right)+\operatorname{Mas}\left(K_{1, i}^{-} \oplus \hat{\mathscr{L}}_{S, 1}, L_{X, t}\right)
\end{aligned}
$$

Thus

$\operatorname{Mas}\left(\mathscr{M}_{5}, \mathscr{N}_{5}\right)+\operatorname{Mas}\left(\mathscr{M}_{10}, \mathscr{N}_{10}\right)=\operatorname{Mas}\left(\mathscr{L}_{S, 1}, L_{X, 1-t}\right)+\operatorname{Mas}\left(K_{1, i}^{-} \oplus \hat{\mathscr{L}}_{S, 1}, L_{X, t}\right)$.

Notice that since $L_{S, 1, t} \circ L_{S, 1,1-t}$ and $L_{X, t} \circ L_{X, 1-t}$ are both homotopic to constant paths, we can compute

$$
\begin{aligned}
& \operatorname{Mas}\left(\mathscr{M}_{5}, \mathscr{N}_{5}\right)+\operatorname{Mas}\left(\mathscr{M}_{10}, \mathscr{N}_{10}\right) \\
& \quad=\operatorname{Mas}\left(\mathscr{L}_{S, 1}, L_{X, 1-t}\right)+\operatorname{Mas}\left(K_{1, i}^{-} \oplus \hat{\mathscr{L}}_{S, 1}, L_{X, t}\right) \\
& \quad=-\operatorname{Mas}\left(L_{S, 1, t}, \mathscr{L}_{X, 1}\right)+\operatorname{Mas}\left(L_{S, 1, t}, K_{\varrho_{1}, i}^{+} \oplus J \hat{\mathscr{L}}_{S, 1}\right) .
\end{aligned}
$$

Just like for the paths 2 and 7 this simplifies to

$$
\begin{aligned}
& \operatorname{Mas}\left(\mathscr{M}_{5}, \mathscr{M}_{5}\right)+\operatorname{Mas}\left(\mathscr{M}_{10}, \mathscr{N}_{10}\right) \\
& =\tau_{\mu}\left(J\left(K_{\varrho_{1}, i}^{-} \oplus \hat{\mathscr{L}}_{S, 1}\right), K_{\varrho_{1}, i}^{+} \oplus J \hat{\mathscr{L}}_{S, 1}, \mathscr{L}_{X, 1}\right)-\tau_{\mu}\left(J \mathscr{L}_{S, 1}, K_{\varrho_{1}, i}^{+} \oplus J \hat{\mathscr{L}}_{S, 1}, \mathscr{L}_{X, 1}\right) \\
& =-\tau_{\mu}\left(J \mathscr{L}_{S, 1}, K_{\varrho_{1}, i}^{+} \oplus J \hat{\mathscr{L}}_{S, 1}, \mathscr{L}_{X, 1}\right) .
\end{aligned}
$$

This shows that $\sum_{i=1}^{9} \operatorname{Mas}\left(\mathscr{M}_{i}, \mathscr{N}_{i}\right)$ simplifies to the desired formula and completes the proof.

\section{Spectral flow on the solid torus}

In our main application discussed in Section [6 we are interested in computing spectral flow of the twisted odd signature operator along a path of $S U(2)$ connections on torus bundles $M$ over $S^{1}$. The main tool is our splitting formula for spectral flow from Theorem 4.3.1, by which we ultimately need to compute 
spectral flow on a solid torus $S \subset M$ and on its complement $M-S$ with certain APS boundary conditions.

While the spectral flow computation on $M-S$ certainly depends on $M$ itself, we will discuss spectral flow on $S$ separately, as it might be interesting and applicable in other settings.

\subsection{Objective}

Let $S=D^{2} \times S^{1}$ be the solid torus and $T=\partial S$ its boundary. Let $A_{\alpha, \beta}$ be the family of connections on $S$ as described in Definition 5.2.1 and let $A_{t}=A_{\alpha_{t}, \beta_{t}}$ be a path of connections on the solid torus $S$, so that $A_{0}$ and $A_{1}$ are flat connections. Boundary conditions are necessary to make the odd signature $D_{A_{t}}$ on $S$ (unbounded) self-adjoint. The Atiyah-Patodi-Singer (APS) boundary conditions $\mathcal{P}_{(\alpha, \beta, \theta)}^{+}(L)$ discussed in Section 3 are suitable for our purpose. The goal is to compute the spectral flow $\operatorname{SF}\left(D_{A_{\varrho t}} ; \mathcal{P}_{\tilde{\varrho}_{t}}^{+}(L)\right)$ of $D_{A_{t}}$ on $S$, where $\varrho=\pi \circ \tilde{\varrho}$.

Since $A_{\alpha, \beta}$ with $\alpha \in \mathbf{Z}$ covers all gauge equivalence classes of flat connections on $S$, the restriction to a path in $A_{\alpha, \beta}$ is suitable for its use in a splitting formula, as we discussed in Section 4.1

\subsection{A smooth family of connections on $S$ parametrized by $\mathbf{R}^{2}$}

We would like to extend the family of flat connections on $T$ given in Definition 3.1.1 to a family $A_{(\alpha, \beta)}$ (smoothly parametrized by $\mathbf{R}^{2}$ ) of connections on $S$, so that

(1) $A_{(\alpha, \beta)}$ is flat for $\alpha \in \mathbf{Z}$,

(2) the restriction of $A_{(\alpha, \beta)}$ to a collar $N(T)$ of $T$ in $S$ is $a_{(\alpha, \beta)}:=-i \alpha d m-$ $i \beta d l \in \mathcal{A}_{N(T)}$.

Notice that $i \beta d l$ makes sense globally as a (flat) connection on $S$ for all $\beta \in \mathbf{R}$, whereas $i \alpha d m$ does not for $\alpha \neq 0$. Thus we can try to construct the family, so that

(3) $A_{(\alpha, \beta)}$ is equal to $-i \beta d l$ for $\alpha=0$ and $\beta \in \mathbf{R}$.

Since $\pi_{2} S U(2)=0$, we can find a gauge transformation $\mathfrak{a}$ on $S$, such that in the collar $N(T)$ of $T$

$$
\begin{aligned}
\left.\mathfrak{a}\right|_{N(T)}: \quad N(T) & \rightarrow S U(2) \\
\left(n, e^{i m}, e^{i l}\right) & \mapsto e^{i m} .
\end{aligned}
$$


The gauge transformation $\mathfrak{a}$ can easily be constructed factoring through $D^{2}$. It will be convenient to fix $\mathfrak{a}\left(n e^{i m}, e^{i l}\right)=q(n) e^{i m}+\sqrt{1-(q(n))^{2}} j$ for a smooth non-decreasing cutoff function $q:[0,1] \rightarrow[0,1]$ with $q(n)=0$ for $n$ near 0 and $q(n)=1$. Since

$$
\begin{aligned}
\left.\mathfrak{a}\right|_{N(T)} \cdot a_{(\alpha, \beta)} & =\mathfrak{a} a_{(\alpha, \beta)} \mathfrak{a}^{-1}+\mathfrak{a} d\left(\mathfrak{a}^{-1}\right)=a_{(\alpha, \beta)}+e^{i m} \frac{\partial}{\partial m} e^{-i m} d m \\
& =a_{(\alpha, \beta)}-i d m=a_{(\alpha+1, \beta)},
\end{aligned}
$$

we get a smooth family of connections $A_{(\alpha, \beta)}:=-\mathfrak{a}^{\alpha} \cdot i \beta d l$ on $S,(\alpha, \beta) \in \mathbf{Z} \times \mathbf{R}$, with the desired property

$$
\left.A_{(\alpha, \beta)}\right|_{N(T)}=\left.\mathfrak{a}^{\alpha}\right|_{N(T)} \cdot a_{(0, \beta)}=a_{(\alpha, \beta)} .
$$

This can be easily extended to a smooth family of connections parametrized by $\mathbf{R}^{2}$ with $\left.A_{(\alpha, \beta)}\right|_{N(T)}=a_{(\alpha, \beta)}$. One possible way is the following:

Definition 5.2.1 Let $\eta: S \rightarrow[0,1]$ be a smooth cutoff function with $\eta(x)=1$ for $x \in N(T)$ and $\eta(x)=0$ near the core of $S$, which may as well factor through $D^{2}$. Let $\left\{\tau_{r}: \mathbf{R} \rightarrow \mathbf{R}\right\}_{r \in \mathbf{Z}}$ be a partition of unity subordinate to $\{(r-1, r+1)\}_{r \in \mathbf{Z}}$. For $(\alpha, \beta) \in \mathbf{R}^{2}$ define the family

$$
A_{(\alpha, \beta)}:=\sum_{r \in \mathbf{Z}} \tau_{r}(\alpha) \mathfrak{a}^{r} \cdot(\eta i(r-\alpha) d m-i \beta d l) .
$$

Notice that $\eta i(r-\alpha) d m-i \beta d l$ is an $S U(2)$ connection on $S$ (in normal form) for $(\alpha, \beta) \in \mathbf{R}^{2}$ and $r \in \mathbf{Z}$. Thus $A_{(\alpha, \beta)}$ is a smooth family of $S U(2)$ connections on $S$. It is easy to check, that this family has the following properties:

(1) $A_{(\alpha, \beta)}=\mathfrak{a} \cdot A_{(\alpha+1, \beta)}$.

(2) $\left.A_{(\alpha, \beta)}\right|_{N(T)}=a_{(\alpha, \beta)}$.

(3) $A_{(\alpha, \beta)}=-\mathfrak{a}^{-\alpha} \cdot i \beta d l$ for $\alpha \in \mathbf{Z}$, which is flat.

\subsection{Computation of spectral flow on the solid torus}

In this section all connections and odd signature operators are considered on the solid torus only. Everything that follows depends on a continuous family of Lagrangians $L_{\alpha, \beta, \theta}$ in $\mathcal{H}^{0+1+2}(T ; \mathbf{R} i)$ parametrized by $\widetilde{\mathbf{R}}^{2}$.

To avoid technical difficulties we are going to assume that $L_{\alpha, \beta, \theta}$ is a family of Lagrangians in $\mathcal{H}^{0+1+2}(T ; \mathbf{R} i)$, which is transverse to $\hat{\mathscr{L}}_{S, A_{(\alpha, \beta)}}$ (see Definition 4.2.2) for all $(\alpha, \beta, \theta) \in \mathbf{Z} \times \mathbf{R} \times\{ \pm i\} \subset \widetilde{\mathbf{R}}^{2}$. Later we will fix a specific family of Lagrangians $L_{\alpha, \beta, \theta}$ in view of the splitting formula in Theorem 4.3 .1 
Definition 5.3.1 Define $\operatorname{SF}(\tilde{\varrho}):=\operatorname{SF}\left(D_{A_{\pi \circ \tilde{\varrho}(t)}}, \mathcal{P}_{\tilde{\varrho}(t)}^{+}(L)\right)$ as a function of paths $\tilde{\varrho}$ in $\widetilde{\mathbf{R}}^{2}$, where $\pi: \widetilde{\mathbf{R}}^{2} \rightarrow \mathbf{R}^{2}$ is the projection mentioned in Definition 3.2.1

Observe that SF is additive under compositions of paths in $\widetilde{\mathbf{R}}^{2}$ and that SF is a homotopy invariant rel endpoints.

Lemma 5.3.2 There is a cohomology class $z \in H^{1}\left(\widetilde{\mathbf{R}}^{2}, \mathbf{Z} \times \mathbf{R} \times\{ \pm i\}\right)$, such that for a path $\tilde{\varrho}$ in $\widetilde{\mathbf{R}}^{2}$ that starts and ends in $\mathbf{Z} \times \mathbf{R} \times\{ \pm i\} \subset \widetilde{\mathbf{R}}^{2}$ the (mod 0$)$ spectral flow equals $z(u)$, where $u:=[\tilde{\varrho}([0,1])] \in H_{1}\left(\widetilde{\mathbf{R}}^{2}, \mathbf{Z} \times \mathbf{R} \times\{ \pm i\}\right)$. Note, that $\mathbf{Z} \times \mathbf{R} \times\{ \pm i\}$ corresponds to the thickened vertical lines in Figure 3 .

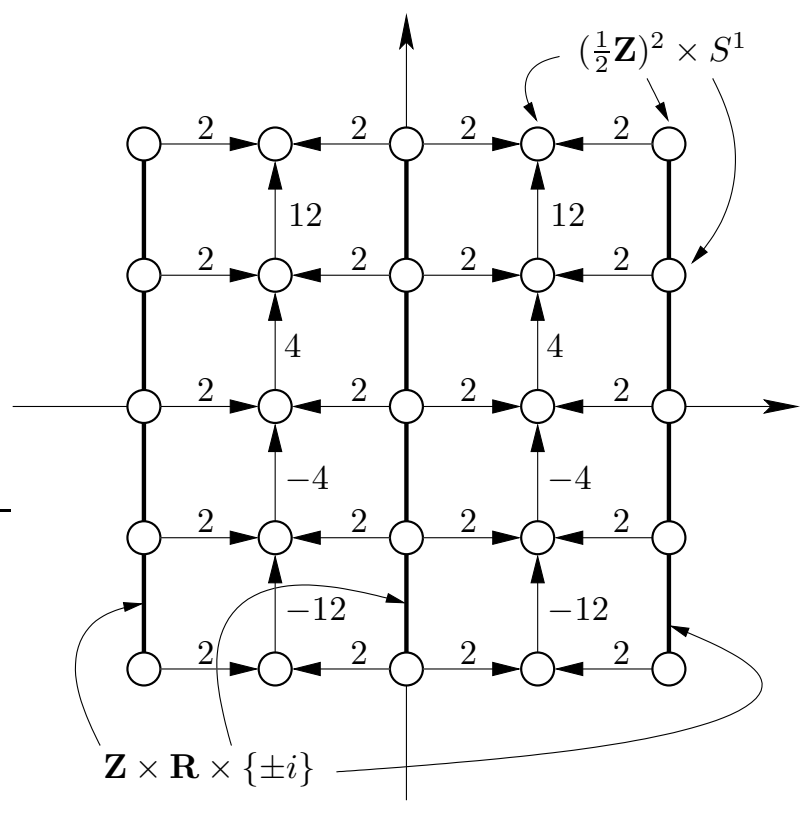

Figure 3: Cycle in $\widetilde{\mathbf{R}}^{2}$

Proof By defining $\zeta\left(\sum_{i} a_{i} \sigma_{i}\right):=\sum_{i} a_{i} \mathrm{SF}\left(\sigma_{i}\right)$ for singular 1-simplices $\sigma_{i}$ and integers $a_{i}$, we get a map $\zeta: S_{1}\left(\widetilde{\mathbf{R}}^{2}\right) \rightarrow \mathbf{Z}$.

Now suppose $(\alpha, \beta) \in \tilde{\varrho}([0,1]) \subset \mathbf{Z} \times \mathbf{R} \times\{ \pm i\}$. Then $A_{\tilde{\varrho}}$ is a path of flat connections on $S$. We have

$$
\operatorname{Ker}\left(H^{1}\left(S ; s u(2)_{\operatorname{hol}\left(A_{\alpha, \beta}\right)}\right) \rightarrow H^{1}\left(T ; s u(2)_{\operatorname{hol}\left(A_{\alpha, \beta}\right)}\right)\right)=0 .
$$

Then Proposition 2.6.3 and $L_{\alpha, \beta} \cap \mathscr{L}_{S, A_{\alpha, \beta}}=0$ imply that $D_{A_{\alpha, \beta}}$ (with boundary conditions $\left.\mathcal{P}_{\alpha, \beta}^{+}(L)\right)$ has no kernel. Furthermore by comparing Theorem 
3.2.2 and Lemma 4.2.1 we see that $K_{\alpha, \beta, \pm i}^{+} \cap \check{\mathscr{L}}_{S, A_{\alpha, \beta}}=0$ when $(\alpha, \beta) \in \mathbf{Z} \times \frac{1}{2} \mathbf{Z}$. This implies that $\operatorname{SF}(\tilde{\varrho})=0$.

Thus $\zeta$ descends to a map $\bar{\zeta}: S_{1}\left(\widetilde{\mathbf{R}}^{2}\right) / S_{1}(\mathbf{Z} \times \mathbf{R} \times\{ \pm i\}) \rightarrow \mathbf{Z}$, that is $\bar{\zeta} \in$ $S^{1}\left(\widetilde{\mathbf{R}}^{2}, \mathbf{Z} \times \mathbf{R} \times\{ \pm i\}\right)$.

It is straightforward to show using the homotopy invariance and the additivity of $\mathrm{SF}$ that $\bar{\zeta}$ is a cocycle. We also observe that by definition a coboundary in $S^{1}\left(\widetilde{\mathbf{R}}^{2}, \mathbf{Z} \times \mathbf{R} \times\{ \pm i\}\right)$ vanishes on paths $\tilde{\varrho}$ in $\widetilde{\mathbf{R}}^{2}$ with endpoints in $\mathbf{Z} \times \mathbf{R} \times\{ \pm i\}$. Thus we have found the desired cohomology class $z=[\bar{\zeta}]$.

Equivalently we may say (by Poincaré duality) that there exists an infinite homology class in $\left.H_{1}\left(\widetilde{\mathbf{R}}^{2}-(\mathbf{Z} \times \mathbf{R} \times\{ \pm i\})\right)\right)$, such that the intersection number with (the homology class representing) the path is equal to the spectral flow.

Fix $L_{\alpha, \beta, \theta}:=J \hat{\mathscr{L}}_{S, A_{(\alpha, \beta)}}=\mathbf{R} i d m \oplus \mathbf{R} i d m \wedge d l=\mathbf{R} i d m \oplus \mathcal{H}^{2}(T ; \mathbf{R} i)$. Our goal is to compute the (infinite) cycle corresponding to the cohomology class $z$ in Lemma 5.3.2, therefore extending [20, Theorem 6.4].

Theorem 5.3.3 If $\tilde{\varrho}$ starts and ends in $\mathbf{Z} \times \mathbf{R} \times\{ \pm i\}$, then $\operatorname{SF}(\tilde{\varrho})$ is given by the intersection number $\tilde{\varrho} \cdot z$ where $z$ is the cycle shown in Figure 3 .

The sign convention for computing the intersection number is determined by the example in Figure 4

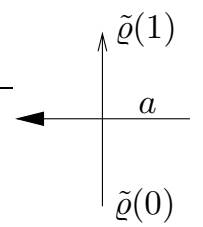

Figure 4: An example with intersection number $a$

The lengthy proof of Theorem 5.3 .3 is deferred to the next section. However, here is a short outline of the proof. Consider a path of connections $A_{t}$ between flat connections on a certain closed manifold $M=S \cup_{T} X$, which restricts to a path $A_{\varrho(t)}$ in our family of connections on $S$ defined in Definition 5.2.1 and $\varrho(t)$ lifts to a path $\tilde{\varrho}(t)$ in $\widetilde{\mathbf{R}}^{2}$. An example of such a path $\tilde{\varrho}(t)$ is shown in Figure 6 . Now consider a particular gauge transformation $g$ on $M$. Suppose that $\varrho^{\prime}=\pi \circ \varrho^{\prime}$ and $\left(g \cdot A_{t}\right)\left|T=A_{\varrho^{\prime}(t)}\right| T$. Then we are going to compare the spectral flow of $g \cdot A_{t}$ with the spectral flow of $B_{t}$ given by

$$
B_{t}= \begin{cases}g \cdot A_{t} & \text { when restricted to X } \\ A_{\varrho^{\prime}(t)} & \text { when restricted to S, }\end{cases}
$$


using some properties of a particular family of gauge transformations as well as Proposition 2.4.1.

By repeating the above process a few times and applying the splitting formula for spectral flow in Theorem 4.3.1 for different paths $\varrho$ we will learn how the chains of the desired (infinite) cycle relate to each other, because the terms of the spectral flow on $X$ cancel. This yields the cycle with three unknowns $a, b$ and $c$. The coefficients in Figure 3 can then be computed using some lens space examples and by applying the splitting formula for spectral flow in Theorem 4.3 .1 once more.

Before we embark on a proof of Theorem 5.3 .3 we adapt some of the definitions and results in [3] to our needs.

Consider the following group of gauge transformations:

$$
\mathcal{G}_{\text {nf }}=\left\{\operatorname{smooth} g: S \rightarrow S U(2) \approx S^{3}|g|_{N(T)}\left(n e^{i m}, e^{i l}\right)=e^{i \alpha m+i \beta l}, \alpha, \beta \in \mathbf{Z}\right\} .
$$

We have $H_{3}\left(S^{3}, S^{1} ; \mathbf{Z}\right)=\mathbf{Z}$ by the long exact sequence of the pair $\left(S^{3}, S^{1}\right)$, and $H_{3}(X, T ; \mathbf{Z})=\mathbf{Z}$ for $\partial X=T$. Thus there is a well-defined degree for maps $(X, T) \rightarrow\left(S^{3}, S^{1}\right)$, particularly for elements of $\mathcal{G}_{\mathrm{nf}}$.

Lemma 5.3.4 (Lemma 4.1, 3]) Let $g, g^{\prime} \in \mathcal{G}_{n f}$. Then $g$ and $g^{\prime}$ are homotopic if and only if $\left.g\right|_{T}=\left.g^{\prime}\right|_{T}$ and $\operatorname{deg}(g)=\operatorname{deg}(h)$.

Consider the following gauge transformations on $S=D^{2} \times S^{1} \rightarrow S U(2)$ :

(1) $\mathfrak{a}\left(n e^{i m}, e^{i l}\right)=q(n) e^{i m}+\sqrt{1-(q(n))^{2}} j$ from Section [5.2.

(2) $\mathfrak{b}\left(n e^{i m}, e^{i l}\right)=e^{i l}$,

(3) $\mathfrak{c}\left(n e^{i m}, e^{i l}\right)$ is a gauge transformation of degree 1 with $\left.\mathfrak{c}\right|_{N(T)} \equiv 1$.

The exact description of these gauge transformations is not relevant for us. However, we will need to exploit some of their properties.

Theorem 5.3.5 (Lemma 4.3, Theorem 4.4, 3]) We have $[\mathfrak{a}, \mathfrak{b}]=\mathfrak{c}^{-2}$ up to homotopy and $\operatorname{deg}\left(\mathfrak{a}^{a} \mathfrak{b}^{b} \mathfrak{c}^{c}\right)=c-a b$.

We are going to use one additional gauge transformation on $S$. We set $\mathfrak{d} \equiv$ $j$. Notice, that even though $\mathfrak{d} \notin \mathcal{G}_{\text {nf }}$, we have $\mathfrak{d} g \mathfrak{d}^{-1} \in \mathcal{G}_{\text {nf }}$ for all $g \in \mathcal{G}_{\text {nf }}$. Furthermore the following observation is noteworthy because we will need it later.

Lemma 5.3.6 $\operatorname{deg}\left(\mathfrak{a} \mathfrak{d} \mathfrak{a} \mathfrak{d}^{-1}\right)=0$. 
Proof We have $H_{3}\left(S^{3}-p, S^{1}\right)=0$ for $p \notin S^{1}$ by the long exact sequence of the pair $\left(S^{3}-p, S^{1}\right)$. Thus, if a map $g:(X, T) \rightarrow\left(S^{3}, S^{1}\right)$ with $\partial X=T$ misses a point $p \notin S^{1}$, then it is a composition of maps

$$
(X, T) \rightarrow\left(S^{3}-p, S^{1}\right) \hookrightarrow\left(S^{3}, S^{1}\right),
$$

and therefore $\operatorname{deg}(g)=0$ by functoriality of $H_{3}$.

Since $\mathfrak{a}$ is homotopic to $\mathfrak{a}^{\prime}\left(n e^{i m}, e^{i l}\right)=n e^{i m}+\sqrt{1-n^{2}} j$, we have for any $\left(n e^{i m}, e^{i l}\right) \in S$

$$
\mathfrak{a}^{\prime} \mathfrak{d} \mathfrak{a}^{\prime} \mathfrak{d}^{-1}\left(n e^{i m}, e^{i l}\right)=2 n^{2}-1+2 n \sqrt{1-n^{2}} e^{i m} j \neq \frac{1}{\sqrt{2}}+\frac{1}{\sqrt{2}} i,
$$

which implies $\operatorname{deg}\left(\mathfrak{a} \mathfrak{d} \mathfrak{a} \mathfrak{d}^{-1}\right)=\operatorname{deg}\left(\mathfrak{a}^{\prime} \mathfrak{d} \mathfrak{a}^{\prime} \mathfrak{d}^{-1}\right)=0$.

The following useful fact follows immediately from the relative Mayer-Vietoris sequence and the long exact sequence of the pairs $(M, T)$ and $\left(S^{3}, S^{1}\right)$.

Lemma 5.3.7 Let $M=S \cup_{T} X$. If $g: M \rightarrow S^{3}$ with $g(T) \subset S^{1}$, then $\operatorname{deg}(g)=\operatorname{deg}\left(\left.g\right|_{S}\right)+\operatorname{deg}\left(\left.g\right|_{X}\right)$.

We want to close this section with a straightforward Maslov index computation (like the one in the proof of Lemma 6.3.1).

Lemma 5.3.8 Let $(\alpha, \beta) \in \mathbf{Z} \times \frac{1}{2} \mathbf{Z}$. Then for small enough $\varepsilon>0$ and varying $t \in[-\varepsilon, \varepsilon]$ we have

$$
\operatorname{Mas}\left(\hat{\mathscr{L}}_{\alpha, \beta}, K_{\alpha, \beta, \theta e^{\pi i t}}^{+}\right)= \begin{cases}2 & \text { if } \theta= \pm 1 \\ 0 & \text { otherwise }\end{cases}
$$

\subsection{Proof of Theorem 5.3 .3}

For simplicity consider the lens space $M=L(4,1)=S \cup_{h} S^{\prime}$, where

$$
h: \partial S=T \rightarrow \partial S^{\prime},\left(e^{i m}, e^{i l}\right) \mapsto\left(e^{i m+i l}, e^{i 4 m+i 3 l}\right) .
$$

Then we have a family of flat connections on $M$ which restricts to noncentral connections on $T$. Figure 5 shows which flat connections of the family of connections $a_{\alpha, \beta}$ on $T$ extend to flat connections on $S$ and $S^{\prime}$. The intersection of the lines corresponding to these flat connections on $T$ correspond to flat connections on $M$. 


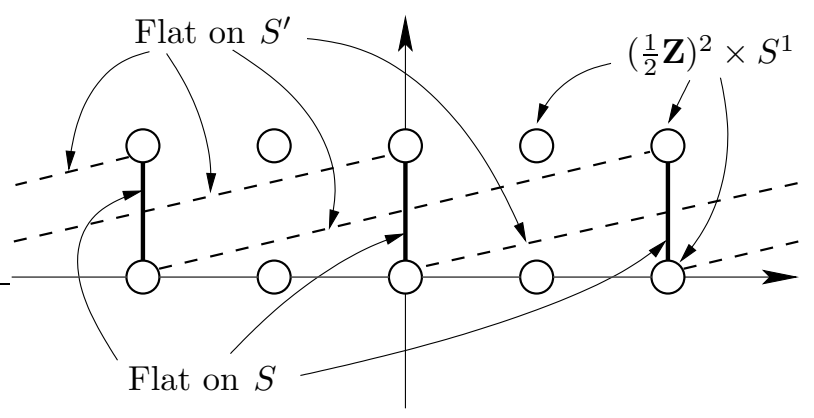

Figure 5: Flat connections $S$ and $S^{\prime}$

Since $\pi_{1}(S U(2))=\pi_{2}(S U(2))=0$, there is no obstruction of extending any $g: S \rightarrow S U(2)$ to $M$. Denote the extensions of $\mathfrak{a}, \mathfrak{b}$ and $\mathfrak{c}$ by $\overline{\mathfrak{a}}, \overline{\mathfrak{b}}$ and $\overline{\mathfrak{c}}$ respectively. We can simply extend $\mathfrak{d}$ to $\overline{\mathfrak{d}}=j$.

Let $\tilde{\varrho}(t)$ be a (straight line) path in $\widetilde{\mathbf{R}}^{2}$ which starts at $\left(0, \frac{1}{4}\right) \in \widetilde{\mathbf{R}}^{2}$ and ends at $\left(1, \frac{1}{4}\right) \in \widetilde{\mathbf{R}}^{2}$ as shown in Figure 6 . Now let $A_{t}$ be a fixed but arbitrary path of connections on $M$, which is flat at the endpoints and equals $A_{\varrho(t)}$ for $\varrho(t)=\tilde{\varrho}(t)$ when restricted to $S$ as in Definition 5.2.1. Consider the gauge transformation $g=\overline{\mathfrak{a}} \overline{\mathfrak{d}}$. Define a path of connections on $M$ by

$$
B_{t}= \begin{cases}g \cdot A_{t} & \text { when restricted to } \mathrm{X} \\ A_{\varrho^{\prime}(t)} & \text { when restricted to } \mathrm{S},\end{cases}
$$

where $\tilde{\varrho}^{\prime}(t)=-\tilde{\varrho}(t)+(1,0)$ and $\varrho^{\prime}(t)=\pi \circ \tilde{\varrho}^{\prime}(t)$. Notice that $\left.A_{\varrho^{\prime}(t)}\right|_{T}=$ $\left.g \cdot A_{\varrho(t)}\right|_{T} \cdot$

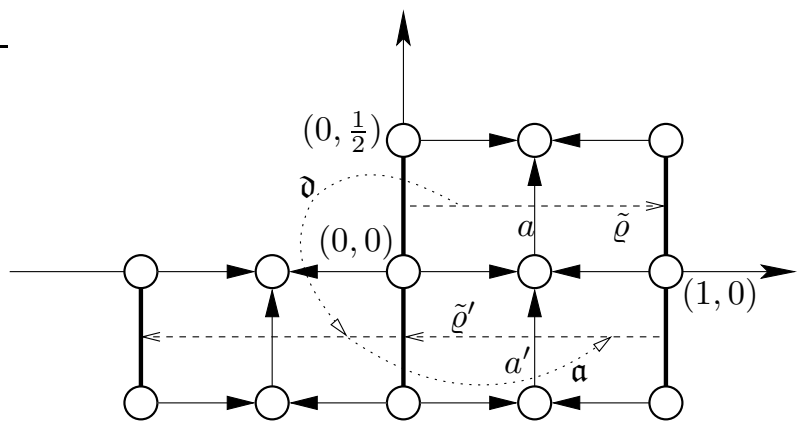

Figure 6: Computing $a^{\prime}=-a$

Let $\mathrm{SF}\left(A_{t}\right):=\operatorname{SF}\left(D_{A_{t}}\right)$. If $\mathcal{P}_{t}$ is a path of APS boundary conditions on $X$ let $\operatorname{SF}_{X}\left(A_{t} ; \mathcal{P}_{t}\right):=\operatorname{SF}\left(\left.D_{A_{t}}\right|_{X} ; \mathcal{P}_{t}\right)$. Occasionally we will also write $\operatorname{SF}\left(A_{0}, A_{1}\right)$ 
instead of $\operatorname{SF}\left(A_{t}\right)$ to emphasize that spectral flow on $M$ only depends on the endpoints. Let us compare $\mathrm{SF}\left(A_{t}\right)$ with $\mathrm{SF}\left(B_{t}\right)$ by utilizing Proposition 2.4.1. By Definition 5.2.1 we have

$$
\left.B_{0}\right|_{S}=\left.\mathfrak{a} \cdot\left(-A_{0}\right)\right|_{S}=\left.\mathfrak{a} \mathfrak{d} \cdot A_{0}\right|_{S}
$$

and

$$
\left.B_{1}\right|_{S}=\left.\mathfrak{d} \cdot A_{0}\right|_{S}=\left.\mathfrak{d} \mathfrak{a}^{-1} \cdot A_{1}\right|_{S}=\left.\mathfrak{d a}^{-1} \mathfrak{d}^{-1} \mathfrak{a}^{-1} \cdot\left(\mathfrak{a} \mathfrak{d} \cdot A_{1}\right)\right|_{S} .
$$

Notice that $\left.B_{1}\right|_{X}=\left.\overline{\mathfrak{a}} \overline{\mathfrak{d}} \cdot A_{1}\right|_{X}$. Consider the gauge transformation

$$
g^{\prime}(x)= \begin{cases}1 & \text { if } x \in X \\ \mathfrak{a} \mathfrak{d} \mathfrak{a d} \mathfrak{d}^{-1}(x) & \text { if } x \in S .\end{cases}
$$

By exploiting the homotopy invariance and the additivity of the spectral flow, Proposition 2.4.1 as well as Lemmas 5.3.7 and 5.3.6 (in this order) we get

$$
\begin{aligned}
\operatorname{SF}\left(A_{t}\right) & =\mathrm{SF}\left(g \cdot A_{t}\right)=\operatorname{SF}\left(\overline{\mathfrak{a}} \overline{\mathfrak{d}} \cdot A_{0}, \overline{\mathfrak{a}} \overline{\mathfrak{d}} \cdot A_{1}\right)=\operatorname{SF}\left(B_{0}, B_{1}\right)+\operatorname{SF}\left(B_{1}, \overline{\mathfrak{a}} \overline{\mathfrak{d}} \cdot A_{1}\right) \\
& =\mathrm{SF}\left(B_{0}, B_{1}\right)+8 \operatorname{deg}\left(g^{\prime}\right)=\operatorname{SF}\left(B_{0}, B_{1}\right)+8 \operatorname{deg}\left(\mathfrak{a} \mathfrak{d} \mathfrak{a} \mathfrak{d}^{-1}\right) \\
& =\mathrm{SF}\left(B_{t}\right)+8 \operatorname{deg}\left(\mathfrak{a} \mathfrak{d} \mathfrak{a d} \mathfrak{d}^{-1}\right)=\operatorname{SF}\left(B_{t}\right) .
\end{aligned}
$$

Let us now apply the splitting formula in Corollary 4.3 .3 to both sides of the equation and observe that for a path of connections $A_{t}$, a gauge transformation $g$ and a path of APS boundary conditions $\mathcal{P}_{t}$ we have

$$
\mathrm{SF}_{X}\left(A_{t} ; \mathcal{P}_{t}\right)=\mathrm{SF}_{X}\left(g \cdot A_{t} ; \operatorname{ad}_{g} \mathcal{P}_{t}\right) .
$$

In particular $\left.A_{t}\right|_{T}=a_{\tilde{\varrho}(t)}$ and $\left.g \cdot A_{t}\right|_{T}=a_{\tilde{\varrho}^{\prime}(t)}$ imply

$$
\operatorname{SF}_{X}\left(A_{t} ; \mathcal{P}_{\tilde{\varrho}(t)}^{-}\left(\hat{\mathscr{L}}_{S}\right)\right)=\operatorname{SF}_{X}\left(g \cdot A_{t} ; \mathcal{P}_{\tilde{\varrho}^{\prime}(t)}^{-}\left(\hat{\mathscr{L}}_{S}\right)\right) .
$$

Thus the spectral flow terms for $X$ vanish, and $a^{\prime}=-a$ in Figure 6 because of

$$
\operatorname{SF}_{S}\left(A_{\varrho(t)} ; \mathcal{P}_{\tilde{\varrho}(t)}^{+}\left(J \hat{\mathscr{L}}_{S}\right)\right)=\operatorname{SF}_{S}\left(A_{\varrho^{\prime}(t)} ; \mathcal{P}_{\tilde{\varrho}^{\prime}(t)}^{+}\left(J \hat{\mathscr{L}}_{S}\right)\right)
$$

Now let $k, l \in \mathbf{Z}$ and consider the straight line path $\tilde{\varrho}(t)$ which starts at $\left(k, \frac{l}{2}+\frac{1}{4}\right)$ and ends at $\left(k, \frac{l}{2}+\frac{5}{4}\right)$, as shown in Figure 7. As before, let $A_{t}$ be a fixed but arbitrary path of connections on $M$, which equals $A_{\varrho(t)}$ for $\varrho(t)=\pi \circ \tilde{\varrho}(t)$ when restricted to $S$ as in definition 5.2.1. Consider the gauge transformation $g=\overline{\mathfrak{b}}$. Using this data, define a path of connections $B_{t}$ on $M$ as in (5.4.1), where $\tilde{\varrho}^{\prime}(t)=\tilde{\varrho}(t)+(0,1)$ and $\varrho(t)=\pi \circ \tilde{\varrho}^{\prime}(t)$. Notice that $\left.A_{\varrho^{\prime}(t)}\right|_{T}=\left.g \cdot A_{\varrho(t)}\right|_{T}$.

We have

$$
\left.B_{0}\right|_{S}=\left.\mathfrak{b} \cdot A_{0}\right|_{S}
$$




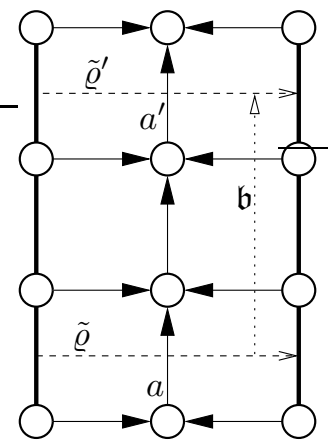

Figure 7: Computing $a^{\prime}=16+a$

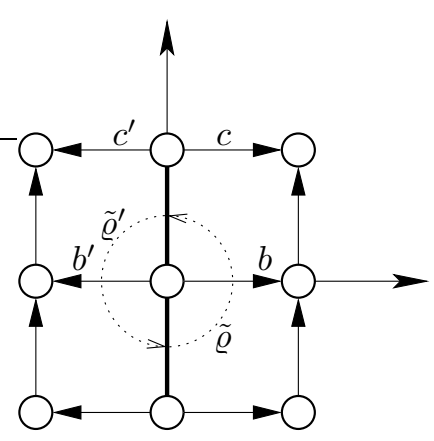

Figure 8: Computing $b^{\prime}=b$

and

$$
\left.B_{1}\right|_{S}=\left.\mathfrak{a} \mathfrak{b} \cdot A_{0}\right|_{S}=\left.\mathfrak{a} \mathfrak{b} \mathfrak{a}^{-1} \mathfrak{b}^{-1} \cdot\left(\mathfrak{b} \mathfrak{a} \cdot A_{0}\right)\right|_{S}=\left.\mathfrak{a} \mathfrak{b} \mathfrak{a}^{-1} \mathfrak{b}^{-1} \cdot\left(\mathfrak{b} \cdot A_{1}\right)\right|_{S} .
$$

Consider the gauge transformation

$$
g^{\prime}(x)= \begin{cases}1 & \text { if } x \in X \\ \left(\mathfrak{a b a}^{-1} \mathfrak{b}^{-1}\right)^{-1}(x) & \text { if } x \in S\end{cases}
$$

Then after invoking Theorem 5.3.5 we get

$$
\begin{aligned}
\mathrm{SF}\left(A_{t}\right) & =\mathrm{SF}\left(g \cdot A_{t}\right)=\mathrm{SF}\left(\overline{\mathfrak{b}} \cdot A_{0}, \overline{\mathfrak{b}} \cdot A_{1}\right)=\mathrm{SF}\left(B_{0}, B_{1}\right)+\mathrm{SF}\left(B_{1}, \overline{\mathfrak{b}} \cdot A_{1}\right) \\
& =\mathrm{SF}\left(B_{0}, B_{1}\right)+\operatorname{deg}\left(g^{\prime}\right)=\operatorname{SF}\left(B_{0}, B_{1}\right)+\operatorname{deg}\left(\left(\mathfrak{a b a} \mathfrak{a}^{-1} \mathfrak{b}^{-1}\right)^{-1}\right) \\
& =\mathrm{SF}\left(B_{t}\right)+\operatorname{deg}\left(\mathfrak{c}^{2}\right)=\operatorname{SF}\left(B_{t}\right)+16 .
\end{aligned}
$$

Application of Corollary 4.3.3 then gives that $a^{\prime}=a+16$ in Figure 7 .

Similarly if for the same path $\varrho$ we consider $\tilde{\varrho}^{\prime}(t)=\tilde{\varrho}(t)+(1,0), \varrho^{\prime}(t)=\pi \circ \tilde{\varrho}^{\prime}(t)$, the gauge transformation $g=\overline{\mathfrak{a}}$, and we define $B_{t}$ as in (5.4.1), then we have $B_{t}=g \cdot A_{t}$ and thus $\mathrm{SF}\left(A_{t}\right)=\mathrm{SF}\left(B_{t}\right)$.

A similar computation gives us the relationship between coefficients for the horizontal simplices. Let $\tilde{\varrho}(t)$ be a path going half around the origin as shown in Figure 8, for example $\tilde{\varrho}(t)=\left(0,0, \frac{1}{4} e^{\pi i\left(t-\frac{1}{2}\right)}\right)$. Consider $\tilde{\varrho}^{\prime}(t)=-\tilde{\varrho}(t)$ and $\varrho^{\prime}(t)=\pi \circ \varrho^{\prime}(t)$. Let $g=\overline{\mathfrak{d}}$ and define $B_{t}$ as in (5.4.1). Since $B_{t}=\overline{\mathfrak{d}} \cdot A_{t}$, we immediately get $\mathrm{SF}\left(A_{t}\right)=\mathrm{SF}\left(B_{t}\right)$. The Corollary 4.3 .3 yields $b=b^{\prime}$ in Figure 8. Similarly, if $g=\overline{\mathfrak{a}}$ or $g=\overline{\mathfrak{b}}$ we have $B_{t}=g \cdot A_{t}$, and we get $c=c^{\prime}$ in Figure 8,

Thus we are left with determining the coefficients $a, b$ and $c$ in our cycle in Figure 9. 


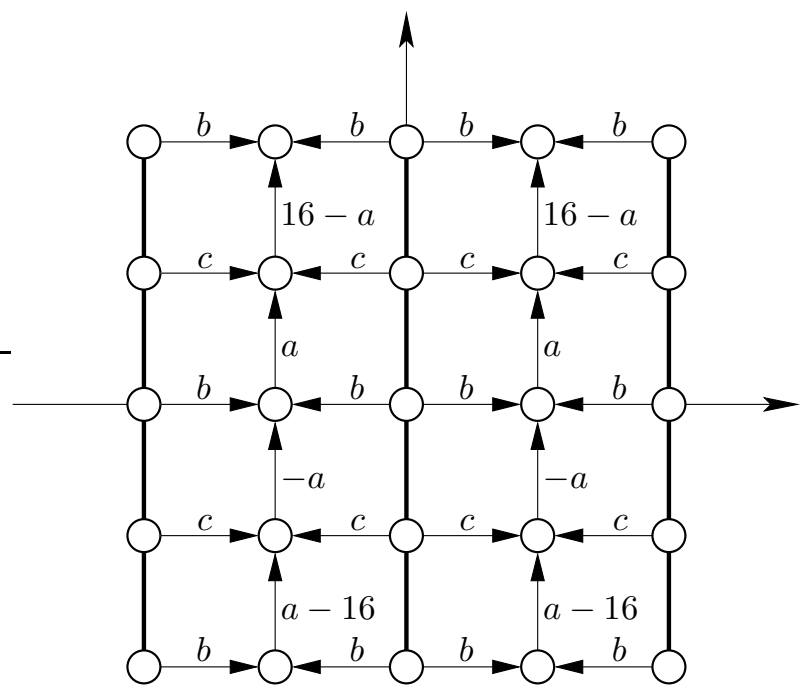

Figure 9: Cycle in $\widetilde{\mathbf{R}}^{2}$

To find the values of $b$ and $c$ we refer to Lemma 5.3.8. For $a$ we consider the 3 -sphere $M$ constructed by gluing two solid tori $S$ and $S^{\prime}$ with the same orientation along the boundary using the orientation reversing homeomorphism on the boundary $\left(e^{i m}, e^{i l}\right) \mapsto\left(e^{i l}, e^{i m}\right)$. For $X=M-S$ we get a similar statement as in Lemma [5.3.8. Then we can employ the splitting formula in Theorem 4.3.1 for some paths $\tilde{\varrho}(t)$ and $\tilde{\varrho}^{\prime}(t)$ with

- $\tilde{\varrho}(0)=(0,0)$,

- $\tilde{\varrho}(t)=\tilde{\varrho}^{\prime}(t)$ for $t \in\left[0, \frac{1}{4}\right] \cup\left[\frac{3}{4}, 1\right]$,

- $\tilde{\varrho}(t)=\left(\frac{1}{2}, 0, e^{4 \pi i t}\right)$ and $\tilde{\varrho}^{\prime}(t)$ constant for $t \in\left[\frac{1}{4}, \frac{3}{4}\right]$.

This yields

$$
\begin{aligned}
0 & =\operatorname{SF}\left(A_{\tilde{\varrho}(t)}\right)-\operatorname{SF}\left(A_{\tilde{\varrho}^{\prime}(t)}\right) \\
& =\operatorname{SF}_{S}\left(A_{\left(\frac{1}{2}, 0, e^{2 \pi i t}\right)} ; \mathcal{P}^{+}\left(J \hat{\mathscr{L}}_{S}\right)\right)+\operatorname{SF}_{X}\left(A_{\left(\frac{1}{2}, 0, e^{2 \pi i t}\right)} ; \mathcal{P}^{-}\left(\hat{\mathscr{L}}_{S}\right)\right) \\
& =(2-a+2-a)+(2+2),
\end{aligned}
$$

and shows that $a$ has to equal 4 .

\section{Spectral flow on a torus bundle over the circle}

We are interested in computing the spectral flow of the odd signature operator coupled to a path of $S U(2)$ connections on a torus bundle over $S^{1}$, because 
it addresses [17, Conjecture 5.8], the missing piece in her work on Witten's 3-manifold invariants [17.

Jeffrey considered a mapping torus $M$ over the torus $T$ and assumed that its monodromy matrix $B$ has $|\operatorname{tr} B| \neq 2$. Her conjecture, based on physical reasoning, implies that the spectral flow of the odd signature operator between irreducible flat $S U(2)$ connections is $0 \bmod 4$, which shall be confirmed in this section.

Lisa Jeffrey's conjecture for the case $G=S U(2)$ needs some interpretation when the trace of the monodromy matrix is zero as well as when one considers representations of $\pi_{1}(M)$ whose restriction to $T$ is central (this is equivalent to the condition in the footnote to [17, Conjecture 5.8] for $G=S U(2))$. Thus we are not able to give a detailed analysis of Lisa Jeffrey's conjecture in this work. However, it will be the subject of future research.

\subsection{Irreducible $S U(2)$ representations of $\pi_{1} M$}

Fix an orientation for $T$. Let $m: T \rightarrow T$ be an orientation preserving homeomorphism, $M=T \times I /(m(x), 1) \sim(x, 0)$ be its mapping torus and fix a base point $(*, 0)$ in $M$. Consider the isomorphism $m_{*}: H_{1}(T ; \mathbf{Z}) \rightarrow H_{1}(T ; \mathbf{Z})$ induced by $m$ on homology, and fix a meridian $x$ and longitude $y$ for $T$ so that $H_{1}(T ; \mathbf{Z}) \cong \pi_{1} T=\langle x, y, \mid[x, y]\rangle \cong \mathbf{Z}^{2}$ and so that $d x \wedge d y$ is in the orientation class, when we consider $x, y \in H_{1}(T ; \mathbf{Z}) \cong H^{1}(T ; \mathbf{Z})$. After identifying $x=\left(\begin{array}{l}1 \\ 0\end{array}\right)$

and $y=\left(\begin{array}{l}0 \\ 1\end{array}\right)$ we can write $m_{*}$ as an unimodular matrix. If $m_{*}(x)=a x+c y$ and $m_{*}(y)=b x+d y$, then the monodromy matrix is

$$
m_{*}=B=\left[\begin{array}{ll}
a & b \\
c & d
\end{array}\right] \in S L_{2}(\mathbf{Z}) .
$$

We will henceforth assume that $|\operatorname{tr} B| \neq 2$. This is equivalent to $\operatorname{det}(B \pm I) \neq 0$ and implies $c \neq 0$ and $b \neq 0$.

The fundamental group of $M$ is an HNN extension of $\pi_{1} T$, where $\tau$ is the loop from $(*, 0)$ to $(m(*), 1)$ :

$$
\pi_{1} M=\left\{x, y, \tau \mid[x, y], \tau x \tau^{-1}=x^{a} y^{c}, \tau y \tau^{-1}=x^{b} y^{d}\right\} .
$$

Recall that we have the identification of $S U(2)$ with the unit quaternions.

Let $\varphi=\left(\varphi_{1}, \varphi_{2}\right) \in \mathbf{R}^{2}$. [20. Proposition 5.5] states that the homomorphism

$$
\begin{aligned}
\rho_{\varphi}:\langle\{x, y, \tau\}\rangle & \rightarrow S U(2) \\
\tau & \mapsto j \\
x & \mapsto e^{2 \pi i \varphi_{1}} \\
y & \mapsto e^{2 \pi i \varphi_{2}}
\end{aligned}
$$


factors through $\pi_{1} M$ if and only if $\varphi(B+I) \in \mathbf{Z}^{2}$. Observe that a representation $\rho_{\varphi}$ is reducible if and only if $\varphi \in\left(\frac{1}{2} \mathbf{Z}\right)^{2}$. Furthermore representations $\rho_{\varphi}$ and $\rho_{\psi}$ are conjugate if and only if $\varphi= \pm \psi+\theta$ for some $\theta \in \mathbf{Z}^{2}$. Together with [20. Corollary 7.2] this implies the following.

Lemma 6.1.1 Any two irreducible $S U(2)$ representations $\rho$ and $\rho^{\prime}$ of $\pi_{1}(M)$ are conjugate to $\rho_{\varphi}$ and $\rho_{\psi}$ respectively so that $\rho_{(1-t) \varphi+t \psi}$ as in (6.1.1) is a path of irreducible $S U(2)$ representations of

$$
\pi_{1}\left(M-N_{\gamma}\right)=\left\langle x, y, \tau \mid[x, y], \tau x^{p} y^{q} \tau^{-1}=x^{p a+q b} y^{p c+q d}\right\rangle,
$$

where $N_{\gamma}$ is a tubular neighborhood of the curve $\gamma=p x+q y$ on $T=T \times\{0\}$ and $(p, q)$ is a relatively prime pair satisfying

$$
(\varphi-\psi)(B+I)\left(\begin{array}{l}
p \\
q
\end{array}\right)=0
$$

Let the longitude $\lambda$ be the curve on $T$ parallel to $\gamma$ and the meridian $\mu$ the curve that bounds a disk in $N_{\gamma}$. It is not hard to see that that $\mu=$ $x^{r a+s b} y^{r c+s d} \tau x^{-r} y^{-s} \tau^{-1}$ and $\lambda=x^{p} y^{q}$ are words representing the meridian and longitude. See [20, Figure 3]. Then for $\varphi=\left(\varphi_{1}, \varphi_{2}\right) \in \mathbf{R}^{2}$ we have

$$
\begin{aligned}
& \rho_{\varphi}(\mu)=e^{2 \pi i \alpha_{\varphi}} \\
& \rho_{\varphi}(\lambda)=e^{2 \pi i \beta_{\varphi}},
\end{aligned}
$$

where $\alpha_{\varphi}:=\varphi(B+I)\left(\begin{array}{l}r \\ s\end{array}\right)$ and $\beta_{\varphi}:=\varphi\left(\begin{array}{l}p \\ q\end{array}\right)$. Thus a representation $\rho_{\varphi}$ of $\pi_{1}(M-$ $N_{\gamma}$ ) restricts to a central representation on the boundary if and only if $\alpha_{\varphi} \in \frac{1}{2} \mathbf{Z}$ and $\beta_{\varphi} \in \frac{1}{2} \mathbf{Z}$.

\subsection{An example}

It is conjectured by Kirk and Klassen in [20, that one can always find some $\varphi$ and $\psi$ so that the entire path $\rho_{t \varphi+(1-t) \psi}$ is noncentral when restricted to $\partial\left(M-N_{\gamma}\right)$. The following example shows that this is false. While Kirk and Klassen's work does not apply here, the present paper does apply to compute the spectral flow.

Consider $B=\left[\begin{array}{ll}5 & 2 \\ 2 & 1\end{array}\right]$. Since $\operatorname{det}(B+I)=8$, conjugacy classes of $S U(2)$ representations of $\pi_{1}(M)$ are uniquely represented by all $\rho_{\varphi}$ with $\varphi \in\left(\frac{1}{8} \mathbf{Z}\right)^{2} \cap$ $\left(\left[0, \frac{1}{2}\right] \times\left[0, \frac{1}{2}\right] \cup\left(\frac{1}{2}, 1\right) \times\left(0, \frac{1}{2}\right)\right)$ for which $\varphi(B+I) \in \mathbf{Z}$. There are only two conjugacy classes of irreducible representations. They are represented by $\rho_{\varphi}$ and $\rho_{\psi}$ for $\varphi=\left(\frac{3}{4}, \frac{1}{4}\right)$ and $\psi=\left(\frac{1}{4}, \frac{1}{4}\right)$. 
Any $\rho_{t \varphi+(1-t) \psi}$ for $t \in[0,1]$ determines a representation of $\pi_{1}\left(M-N_{\gamma}\right)$, where $\gamma=p x+q y$ is such that $(p, q)$ is a relatively prime pair satisfying equation (6.1.2). In this case we have $\varphi(B+I)=(5,2)$ and $\psi(B+I)=(2,1)$. Thus we can choose $(p, q)= \pm(1,-3)$. Conjugates $\rho_{\varphi^{\prime}}$ and $\rho_{\psi^{\prime}}$ of $\rho_{\varphi}$ and $\rho_{\psi}$ give us a different $(p, q)$. For example if we choose $\varphi^{\prime}=\varphi$ and $\psi^{\prime}=-\psi$, then $(p, q)= \pm(3,-7)$.

Proposition 6.2.1 Let $B=\left[\begin{array}{ll}5 & 2 \\ 2 & 1\end{array}\right], \varphi=\left(\frac{3}{4}, \frac{1}{4}\right)$ and $\psi=\left(\frac{1}{4}, \frac{1}{4}\right)$. Let $\varphi^{\prime}= \pm \varphi+\eta$ and $\psi^{\prime}= \pm \psi+\theta$ for $\eta, \theta \in \mathbf{Z}^{2}$ and $\gamma=p x+q y$ a knot satisfying 6.1.2 for $\varphi^{\prime}$ and $\psi^{\prime}$. Then both $\rho_{\varphi^{\prime}}$ and $\rho_{\psi^{\prime}}$ are central when restricted to $\partial\left(M-N_{\gamma}\right)$.

Proof We have $(\varphi-\psi)(B+I)=(3,1)$ and $(\varphi+\psi)(B+I)=(7,3)$. Furthermore for $\theta=\left(\theta_{1}, \theta_{2}\right) \in \mathbf{Z}^{2}$ we get $\theta(B+I)=\left(6 \theta_{1}+2 \theta_{2}, 2 \theta_{1}+2 \theta_{2}\right)=$ $2\left(3 \theta_{1}+\theta_{2}, \theta_{1}+\theta_{2}\right)$. Similarly $\eta(B+I)=2\left(3 \eta_{1}+\eta_{2}, \eta_{1}+\eta_{2}\right)$. It follows that $\left(\varphi^{\prime}-\psi^{\prime}\right)(B+I)$ is a pair of odd integers. Since $(p, q)$ are required to be relatively prime, one of them must be odd. Since $\left(\varphi^{\prime}-\psi^{\prime}\right)(B+I)\left(\begin{array}{l}p \\ q\end{array}\right)=0$, both $p$ and $q$ must be odd. This implies that $\beta_{\varphi^{\prime}}$ and $\beta_{\psi^{\prime}}$ are half integers. Since $\alpha_{\varphi^{\prime}}$ and $\alpha_{\psi^{\prime}}$ are also integers, $\left.\rho_{\varphi^{\prime}}\right|_{\partial\left(M-N_{\gamma}\right)}$ and $\left.\rho_{\psi^{\prime}}\right|_{\partial\left(M-N_{\gamma}\right)}$ are central.

A similar example is given by $B=\left(\begin{array}{ll}3 & 4 \\ 2 & 3\end{array}\right)$ with $\varphi=\left(\frac{1}{4}, 0\right), \psi=\left(\frac{1}{4}, \frac{1}{2}\right)$.

In addition to the above examples there are also paths with representations in the interior which are central on $\partial\left(M-N_{\gamma}\right)$. The example

$$
B=\left(\begin{array}{cc}
9 & 4 \\
2 & 1
\end{array}\right) \text { with }\left\{\varphi=\left(\frac{1}{6}, \frac{1}{6}\right), \psi=\left(\frac{2}{3}, \frac{1}{6}\right)\right\} \text { or }\left\{\varphi=\left(\frac{5}{6}, \frac{1}{3}\right), \psi=\left(\frac{1}{3}, \frac{1}{3}\right)\right\}
$$

are particularly interesting, because all conjugate choices of $\rho_{\varphi}$ and $\rho_{\psi}$ seem to still have representations in the interior which are central on the boundary, though no proof has been found.

\subsection{Computation of the spectral flow}

We compute the mod 4 spectral flow of the odd signature operator on $M$ coupled to a path of $S U(2)$ connections $A_{t}$, where $A_{0}$ and $A_{1}$ are flat and irreducible. We want to apply the splitting formula in Theorem 4.3.1. By Lemma 6.1.1 we may assume that $\rho_{\varphi_{0}}=\operatorname{hol}\left(A_{0}\right)$ and $\rho_{\varphi_{1}}=\operatorname{hol}\left(A_{1}\right)$, and that $\rho_{\varphi_{t}}$ with $\varphi_{t}:=(1-t) \varphi_{0}+t \varphi_{1}$ for $t \in[0,1]$ is a path of irreducible representations 
of $\pi_{1}\left(M-N_{\gamma}\right)$ for some curve $\gamma$. The holonomy maps flat connections on $M-N_{\gamma}$ to representations of $\pi_{1}\left(M-N_{\gamma}\right)$. By [14, Theorem 4.1] there is local splitting of this holonomy map. Thus $\rho_{\varphi_{t}}$ lifts to a path of irreducible connections on $X:=M-N_{\gamma}$ with holonomy $\rho_{\varphi_{t}}$, which extends to a path $A_{t}$ of connections on all of $M$ via the family of connections $A_{\alpha, \beta}$ on $S:=N_{\gamma}$ given in Definition 5.2.1. Notice that $\left.A_{t}\right|_{S}=A_{\alpha_{t}, \beta_{t}}$ where $\left(\alpha_{t}, \beta_{t}\right):=\left(\alpha_{\varphi_{t}}, \beta_{\varphi_{t}}\right)$. The slope of $\left(\alpha_{t}, \beta_{t}\right)$ corresponds to $\theta^{\star}:=\frac{\left(\alpha_{1}-\alpha_{0}\right)+\left(\beta_{1}-\beta_{0}\right) i}{\sqrt{\left(\alpha_{1}-\alpha_{0}\right)^{2}+\left(\beta_{1}-\beta_{0}\right)^{2}}} \in S^{1}$.

Kirk and Klassen computed the spectral flow of $D_{A_{t}} \bmod 4$ [20, Theorem 7.5], when the representations $\rho_{\varphi_{t}}=\operatorname{hol}\left(\left.A_{t}\right|_{X}\right)$ are noncentral for all $t$ when restricted to $T=\partial X$. Recall that $\left.\rho_{\varphi_{t}}\right|_{T}$ is central if and only if $\left(\alpha_{t}, \beta_{t}\right) \in\left(\frac{1}{2} \mathbf{Z}\right)^{2}$.

Let us reparametrize $\varphi_{t}$, so that $\varrho_{t}:=\left(\alpha_{t}, \beta_{t}\right)$ lifts to a path $\varrho_{t}$ in $\widetilde{\mathbf{R}}^{2}$. The spectral flow on the solid torus in Theorem 4.3.1 has been computed in Theorem 5.3.3. Therefore, we focus on the spectral flow on $X$ and the Maslov triple indices in Theorem 4.3.1.

We apply Proposition 2.6.1 to the cohomology computations in 20, Lemma 7.7] to compute the scattering Lagrangian $\mathscr{L}_{X, t}$ of $A_{t}$ on $X$ when $\left.\rho_{\varphi_{t}}\right|_{T}$ is noncentral. Analogous computations yield $\mathscr{L}_{X, t}$ when $\left.\rho_{\varphi_{t}}\right|_{T}$ is central but $\rho_{\varphi_{t}}$ is irreducible. We see that the scattering Lagrangian on $X$ splits into the $\mathbf{R} i$ and $\mathbf{C} j$ part $\mathscr{L}_{X, t}=\hat{\mathscr{L}}_{X, t} \oplus \check{\mathscr{L}}_{X, t}$, just like the scattering Lagrangian on $S$. We have

$$
\hat{\mathscr{L}}_{X, t}=\operatorname{span}\{i(\operatorname{det}(B+I) d m-c d l), i d m \wedge d l\}
$$

and

$$
\check{\mathscr{L}}_{X, t}= \begin{cases}0 & \text { if }\left(\alpha_{t}, \beta_{t}\right) \notin\left(\frac{1}{2} \mathbf{Z}\right)^{2} \\ e^{i\left(2 \alpha_{t} m+2 \beta_{t} l\right)}(\mathbf{C} j d m \oplus \mathbf{C} j d m \wedge d l) & \text { if }\left(\alpha_{t}, \beta_{t}\right) \in\left(\frac{1}{2} \mathbf{Z}\right)^{2} .\end{cases}
$$

Thus, by the additivity of the Maslov triple index, the Maslov triple indices in Theorem 4.3.1 reduce to $\tau\left(K_{\alpha_{0}, \beta_{0}, i}^{-}, \check{\mathscr{L}}_{S, 0}, \check{\mathscr{L}}_{X, 0}\right)$ and $\tau\left(K_{\alpha_{1}, \beta_{1}, i}^{-}, \check{\mathscr{L}}_{S, 1}, \check{\mathscr{L}}_{X, 1}\right)$.

Lemma 6.3.1 If $\left(\alpha_{t^{\star}}, \beta_{t^{\star}}\right) \in\left(\frac{1}{2} \mathbf{Z}\right)^{2}$ for some $t^{\star} \in[0,1]$, then for small enough $\varepsilon>0$ and varying $t \in[-\varepsilon, \varepsilon]$ we have

$$
\operatorname{Mas}\left(K_{\left(\alpha_{t^{\star}}, \beta_{t^{\star}}, \theta e^{t i}\right)}^{-}, \check{\mathscr{L}}_{X, A_{t^{\star}}}\right)= \begin{cases}2 & \text { if } \theta= \pm 1 \\ 0 & \text { otherwise }\end{cases}
$$

Proof By Theorem 3.2 .2 we observe

$$
\operatorname{dim}\left(K_{\left(\alpha_{t^{\star}}, \beta_{t^{\star}}, \theta\right)}^{-} \cap \check{\mathscr{L}}_{X, A_{t^{\star}}}\right)= \begin{cases}2 & \text { if } \theta= \pm 1, \\ 0 & \text { otherwise. }\end{cases}
$$


Consider the case $\theta=1$. To make notation simpler assume $\left(\alpha_{t^{\star}}, \beta_{t^{\star}}\right)=(0,0)$. Then $e^{i\left(2 \alpha_{t^{\star}} m+2 \beta_{t^{\star}} l\right)}=1$. Notice that $K_{(0,0,1)}^{-}$and $\check{\mathscr{L}}_{X, A_{t^{\star}}}$ intersect in

$$
\operatorname{span}\{j d m \wedge d l-k d m, k d m \wedge d l+j d m\} .
$$

Consider the constant path $\tilde{L}:=\operatorname{span}\{k d m, j d m \wedge d l\}$ and the path

$L_{t}=\operatorname{span}\{j d m \wedge d l-k(\cos t d m+\sin t d l), j-k(\cos t d l-\sin t d m)\} \subset K_{\left(\alpha, \beta, e^{t i}\right.}^{-}$

of 2-dimensional Lagrangians in the symplectic subspace

$$
\operatorname{span}\{j, k d m, k d l, j d m \wedge d l\} \subset \mathcal{H}^{0+1+2}(M ; \mathbf{C} j),
$$

parametrized by $t \in[-\varepsilon, \varepsilon]$. These intersect at $t=0$ in $\operatorname{span}\{j d m \wedge d l-k d m\}$. We compute

$$
\begin{aligned}
& \operatorname{Mas}\left(L_{t}, \tilde{L}\right) \\
& \left.=\operatorname{Mas}\left(\operatorname{span}\left\{j d m \wedge d l-\left(e^{J t} k d m\right), j-\left(e^{J t} k d l\right)\right)\right\}, \tilde{L}\right) \\
& =\operatorname{Mas}\left(\mathscr{L}_{1} * e^{J t} \operatorname{span}\{j d m \wedge d l-k d m, j-k d l\} * \mathscr{L}_{2}, \tilde{L}\right) \\
& =\operatorname{Mas}\left(e^{J t} \operatorname{span}\{j d m \wedge d l-k d m, j-k d l\}, \tilde{L}\right) \\
& =1,
\end{aligned}
$$

where $*$ denotes composition of paths, and the paths

$$
\begin{aligned}
\mathscr{L}_{1}(t) & :=\operatorname{span}\left\{e^{-J t} j d m \wedge d l-e^{-J \varepsilon} k d m, e^{-J t} j-e^{-J \varepsilon} k d l\right\}, \\
\mathscr{L}_{2}(t) & :=\mathscr{L}_{1}(t-\varepsilon)
\end{aligned}
$$

are parametrized by $t \in[0, \varepsilon]$. Observe that $\operatorname{Mas}\left(\mathscr{L}_{i}, \tilde{L}\right)=0$ since $k d l \perp \tilde{L}$.

Now for the orthogonal complement in $\mathcal{H}^{0+1+2}(M ; \mathbf{C} j)$ we have

$$
\operatorname{Mas}\left(L_{t}^{\perp}, \tilde{L}^{\perp}\right)=\operatorname{Mas}\left(J L_{t}, J \tilde{L}\right)=\operatorname{Mas}\left(L_{t}, \tilde{L}\right)=1 .
$$

Thus

$$
\operatorname{Mas}\left(K_{\left(0,0, e^{t i}\right)}^{-}, \check{\mathscr{L}}_{X, A_{t^{\star}}}\right)=\operatorname{Mas}\left(L_{t} \oplus L_{t}^{\perp}, \tilde{L} \oplus \tilde{L}^{\perp}\right)=2 .
$$

A similar computation proves the case $\theta=-1$.

This implies together with Lemma 5.3.8 and the fact that $K_{\alpha, \beta,-\theta}^{-}=K_{\alpha, \beta, \theta}^{+}$, that the Maslov triple indices are either 0 or 4 each.

Now we analyze the spectral flow on $X$, when $\varrho=\left(\alpha_{t}, \beta_{t}\right)$ passes through the half integer lattice. In the following proposition we compute the spectral flow for its lift $\tilde{\varrho}$ in $\widetilde{\mathbf{R}}^{2}$ locally around the half integer lattice. 
Proposition 6.3.2 Let $[a, b]$ be a maximal interval with $\varrho([a, b])=(\alpha, \beta) \in$ $\left(\frac{1}{2} \mathbf{Z}\right)^{2}$. Then for small enough $\varepsilon>0$ and varying $t \in[-\varepsilon-a, b+\varepsilon]$ the spectral flow

$$
\operatorname{SF}\left(\left.D_{A_{t}}\right|_{X} ; \mathcal{P}_{\varrho_{t}}^{-}\left(\hat{\mathscr{L}}_{S, t}\right)\right)
$$

is twice the algebraic intersection number of $\left.\tilde{\varrho}\right|_{[a, b]}$ with $\{(\alpha, \beta, \pm 1)\} \subset \widetilde{\mathbf{R}}^{2}$.

Proof Consider $\left(\alpha_{t}, \beta_{t}\right) \in\left(\frac{1}{2} \mathbf{Z}\right)^{2}$. The computation $H^{1}\left(X ; s u(2)_{\rho_{\varphi_{t}}}\right) \cong \mathbf{R}^{3}$ is the same as in the proof of [20, Lemma 7.7]. For $\left(\alpha_{t}, \beta_{t}\right) \notin\left(\frac{1}{2} \mathbf{Z}\right)^{2}$ we have by our computation of $\mathscr{L}_{X, t}$, Proposition 2.6.1 and the long exact sequence of of the pair $(X, T)$ that $\operatorname{Im}\left(H^{1}\left(X, T ; s u(2)_{\rho_{\varphi_{t}}}\right) \rightarrow H^{1}\left(X ; s u(2)_{\rho_{\varphi_{t}}}\right)\right)=0$. Together with the computations from [20, Lemma 7.7] we get

$$
\operatorname{Im}\left(H^{1}\left(X, T ; s u(2)_{\rho_{\varphi_{t}}}\right) \rightarrow H^{1}\left(X ; s u(2)_{\rho_{\varphi_{t}}}\right)\right)= \begin{cases}0 & \text { if } \alpha_{t} \in \frac{1}{2} \mathbf{Z} \text { or } \beta_{t} \notin \frac{1}{2} \mathbf{Z} \\ 2 & \text { otherwise. }\end{cases}
$$

Then by Proposition 2.6.3 $D_{A_{t}}$ has non-resonance level 0 on $X$ when $\alpha_{\varphi} \in \frac{1}{2} \mathbf{Z}$ or $\beta_{\varphi} \notin \frac{1}{2} \mathbf{Z}$.

Recall that by $|\operatorname{tr} B| \neq 2$ we have $\operatorname{det}(B+I) \neq 0$ and $c \neq 0$. Thus $\hat{\mathscr{L}}_{X, t}$ is transverse to $\hat{\mathscr{L}}_{S, t}$ for all $t$ and $\theta^{\star} \neq \pm 1$, because the 1 -forms of $\hat{\mathscr{L}}_{X, t}$ make up the tangent space to the path $i \alpha_{t} d m+i \beta_{t} d l$ by [20, Lemma 6.3].

We have $\tilde{\varrho}(a)=\left(\alpha, \beta,-\theta^{\star}\right)$ and $\tilde{\varrho}(b)=\left(\alpha, \beta, \theta^{\star}\right)$. Since $\theta^{\star} \neq \pm 1$, we can find an $\varepsilon>0$ with $\beta_{t} \notin \frac{1}{2} \mathbf{Z}$ for $t \in[-\varepsilon+a, a) \cup(b, b+\varepsilon]$. Then $D_{A_{t}}$ has nonresonance level 0 , and by Proposition 2.6.3 we get for $t \in[-\varepsilon+a, a) \cup(b, b+\varepsilon]$

$$
\Lambda_{X, t} \cap\left(P_{t}^{-} \oplus \mathscr{L}_{S, t}\right) \cong \hat{\mathscr{L}}_{X, t} \cap \mathscr{L}_{S, t}=0 .
$$

For $t=a$ and $t=b$ we also have non-resonance level 0 . Since $\theta^{\star} \neq \pm 1$ we get

$$
\Lambda_{X, t} \cap \mathcal{P}_{\alpha, \beta, \pm \theta^{\star}}^{-} \cong\left(\hat{\mathscr{L}}_{X, t} \oplus \check{\mathscr{L}}_{X, t}\right) \cap\left(\hat{\mathscr{L}}_{S, t} \oplus K_{\alpha, \beta, \pm \theta^{\star}}^{-}\right)=0 .
$$

This implies that for $\left.\varrho\right|_{[-\varepsilon+a, a]}$ and $\left.\varrho\right|_{[b, b+\varepsilon]}$ the spectral flow on $X$ vanishes.

Since we have non-resonance level 0 on $X$ for all $t \in[a, b]$ we have $\Lambda_{X, t}^{\infty}=$ $P_{a_{t}}^{+} \oplus \mathscr{L}_{X, t}$. By Proposition 2.6.5 and since the Maslov index is invariant under a homotopy of paths of Lagragians which preserves the dimension of the intersections at the endpoints, we get for varying $t \in[a, b]$

$$
\begin{aligned}
& \operatorname{Mas}\left(\mathcal{P}_{\varrho_{t}}^{-}\left(\hat{\mathscr{L}}_{S, t^{\star}}\right), \Lambda_{X, t}\right) \\
& =\operatorname{Mas}\left(\mathcal{P}_{\tilde{\varrho}_{t}}^{-}\left(\hat{\mathscr{L}}_{S}\right), P_{a_{t}}^{+} \oplus \mathscr{L}_{X, t}\right) \\
& =\operatorname{Mas}\left(P_{a_{t}}^{-}, P_{a_{t}}^{+}\right)+\operatorname{Mas}\left(K_{\tilde{\varrho}_{t}}, \check{\mathscr{L}}_{X, t}\right)+\operatorname{Mas}\left(\hat{\mathscr{L}}_{S, t}, \hat{\mathscr{L}}_{X, t}\right)
\end{aligned}
$$

The first and last summand vanish, the second is determined by Lemma 6.3.1. This completes the computation. 
The above proposition together with the computation in the proof of 20 , Lemma 7.7], that the spectral flow on $X$ picks up $2 \bmod 4$, whenever $\beta_{t} \in \frac{1}{2} \mathbf{Z}$ and $\alpha_{t} \notin \frac{1}{2} \mathbf{Z}$, implies that

$$
\operatorname{SF}\left(\left.D_{A_{t}}\right|_{S} ; \mathcal{P}_{\tilde{\varrho}_{t}}^{+}\left(J \hat{\mathscr{L}}_{S, t}\right)\right)+\operatorname{SF}\left(\left.D_{A_{t}}\right|_{X} ; \mathcal{P}_{\tilde{\varrho}_{t}}^{-}\left(\hat{\mathscr{L}}_{S, t}\right)\right)
$$

is a multiple of 4 . We summarize.

Theorem 6.3.3 Let $A_{t}$ be a path of $S U(2)$ connections on a torus bundle over $S^{1}$, where $A_{0}$ and $A_{1}$ are flat and irreducible. Then $\operatorname{SF}\left(D_{A_{t}}\right) \equiv 0 \bmod 4$.

\section{References}

[1] M F Atiyah, V K Patodi, I M Singer, Spectral asymmetry and Riemannian geometry. I, Math. Proc. Cambridge Philos. Soc. 77 (1975) 43-69 MathReview

[2] D Bleecker, B Booss-Bavnbek, Spectral invariants of operators of Dirac type on partitioned manifolds, from: "Aspects of boundary problems in analysis and geometry", Oper. Theory Adv. Appl. 151, Birkhäuser, Basel (2004) 1-130 MathReview

[3] H U Boden, C M Herald, P A Kirk, E P Klassen, Gauge theoretic invariants of Dehn surgeries on knots, Geom. Topol. 5 (2001) 143-226 MathReview

[4] B Booss-Bavnbek, M Lesch, J Phillips, Unbounded Fredholm operators and spectral flow, Canad. J. Math. 57 (2005) 225-250 MathReview

[5] B Booß-Bavnbek, KP Wojciechowski, Elliptic boundary problems for Dirac operators, Mathematics: Theory \& Applications, Birkhäuser Boston Inc. Boston, MA (1993) MathReview

[6] U Bunke, On the gluing problem for the $\eta$-invariant, J. Differential Geom. 41 (1995) 397-448 MathReview

[7] S E Cappell, R Lee, E Y Miller, On the Maslov index, Comm. Pure Appl. Math. 47 (1994) 121-186 MathReview

[8] S E Cappell, R Lee, E Y Miller, Self-adjoint elliptic operators and manifold decompositions. I. Low eigenmodes and stretching, Comm. Pure Appl. Math. 49 (1996) 825-866 MathReview

[9] A M Daniel Maslov index, symplectic reduction in a symplectic Hilbert space and a splitting formula for spectral flow, $\mathrm{PhD}$ thesis, Indiana University, Bloomington, (1997)

[10] M Daniel, An extension of a theorem of Nicolaescu on spectral flow and the Maslov index, Proc. Amer. Math. Soc. 128 (2000) 611-619 MathReview

[11] M Daniel, P Kirk, A general splitting formula for the spectral flow, Michigan Math. J. 46 (1999) 589-617 MathReview 
[12] J F Davis, P Kirk, Lecture notes in algebraic topology, Graduate Studies in Mathematics 35, American Mathematical Society, Providence, RI (2001) MathReview

[13] S K Donaldson, P B Kronheimer, The geometry of four-manifolds, Oxford Mathematical Monographs, The Clarendon Press Oxford University Press, New York (1990) MathReview

[14] B Fine, P Kirk, E Klassen, A local analytic splitting of the holonomy map on flat connections, Math. Ann. 299 (1994) 171-189 MathReview

[15] B Himpel A Splitting Formula for Spectral flow on closed 3-manifolds, PhD thesis, Indiana University, Bloomington (2004)

[16] B Himpel, P Kirk, M Lesch, Calderón projector for the Hessian of the perturbed Chern-Simons function on a 3-manifold with boundary, Proc. London Math. Soc. (3) 89 (2004) 241-272 MathReview

[17] L C Jeffrey, Chern-Simons-Witten invariants of lens spaces and torus bundles, and the semiclassical approximation, Comm. Math. Phys. 147 (1992) 563-604 MathReview

[18] T Kato Perturbation Theory for Linear Operators, reprint of the 1980 edition, Classics in Mathematics, Springer-Verlag (1995) MathReview

[19] P A Kirk, E P Klassen, Chern-Simons invariants of 3-manifolds and representation spaces of knot groups, Math. Ann. 287 (1990) 343-367 MathReview

[20] P Kirk, E Klassen, Computing spectral flow via cup products, J. Differential Geom. 40 (1994) 505-562 MathReview

[21] P Kirk, E Klassen, Continuity and analyticity of families of self-adjoint Dirac operators on a manifold with boundary, Illinois J. Math. 42 (1998) 123-138 MathReview

[22] P Kirk, E Klassen, D Ruberman, Splitting the spectral flow and the Alexander matrix, Comment. Math. Helv. 69 (1994) 375-416 MathReview

[23] P Kirk, M Lesch, The $\eta$-invariant, Maslov index, and spectral flow for Diractype operators on manifolds with boundary, Forum Math. 16 (2004) 553-629 MathReview

[24] L I Nicolaescu, The Maslov index, the spectral flow, and decompositions of manifolds, Duke Math. J. 80 (1995) 485-533 MathReview

[25] T Ohtsuki, Problems on invariants of knots and 3-manifolds, from: "Invariants of knots and 3-manifolds (Kyoto, 2001)", Geom. Topol. Monogr. 4 (2002) 377-572 MathReview

[26] E Witten, Quantum field theory and the Jones polynomial, Comm. Math. Phys. 121 (1989) 351-399 MathReview

[27] K P Wojciechowski, The additivity of the $\eta$-invariant: the case of an invertible tangential operator, Houston J. Math. 20 (1994) 603-621 MathReview 